\title{
Special feature of COVID-19 in FMODB: Fragment molecular orbital calculations and interaction energy analysis of SARS-CoV-2 related proteins
}

Kaori Fukuzawa ${ }^{\mathrm{a}, \mathrm{b}^{*}}$, Koichiro Kato ${ }^{\mathrm{c}, \mathrm{d}^{*}}$, Chiduru Watanabe $\mathrm{e}^{\mathrm{e}, \mathrm{f}^{*}}$, Yusuke Kawashima ${ }^{\mathrm{a}}$, Yuma Handa ${ }^{\mathrm{a}}$, Ami Yamamoto $^{\text {a }}$, Kazuki Watanabe ${ }^{\mathrm{g}, \mathrm{h}}$, Tatsuya Ohyama ${ }^{\mathrm{e}, \mathrm{i}}$, Kikuko Kamisaka ${ }^{\mathrm{e}}$, Daisuke Takaya ${ }^{\mathrm{e}}$, Teruki Honma $^{\mathrm{e}^{*}}$

a. Department of Physical Chemistry, School of Pharmacy and Pharmaceutical Sciences, Hoshi University, 2-4-41 Ebara, Shinagawa-ku, Tokyo 142-8501, Japan

b. Department of Biomolecular Engineering, Graduate School of Engineering, Tohoku University, 6-6-11 Aoba, Aramaki, Aoba-ku, Sendai 980-8579, Japan

c. Department of Applied Chemistry, Graduate School of Engineering, Kyushu University, 744 Motooka, Nishi-ku, Fukuoka 819-0395, Japan

d. Center for Molecular Systems (CMS), Kyushu University, 744 Motooka, Nishi-ku, Fukuoka 8190395, Japan

e. RIKEN Center for Biosystems Dynamics Research, 1-7-22 Suehiro-cho, Tsurumi-ku, Yokohama, Kanagawa, 230-0045, Japan

f. JST PRESTO, 4-1-8, Honcho, Kawaguchi, Saitama 332-0012, Japan

g. Graduate School of Pharmaceutical Sciences, Osaka University, 1-6 Yamadaoka, Suita City, Osaka 565-0871, Japan

h. Graduate School of Pharmaceutical Sciences, Chiba University, 1-8-1 Inohana, Chuo-ku, Chiba 260-8675, Japan

i. Frontier Institute for Biomolecular Engineering Research (FIBER), Konan University, 7-1-20, Minatojima-minamimachi, Chuo-ku, Kobe 650-0047, Japan

*Corresponding author. Email: k-fukuzawa@hoshi.ac.jp (K. Fukuzawa), kato.koichiro.957@m.kyushu-u.ac.jp (K. Kato), chiduru.watanabe@riken.jp (C. Watanabe), honma.teruki@riken.jp (T. Honma)

\section{Abstract}

SARS-CoV-2 is the causative agent of coronavirus(known as COVID-19), the virus causing the current pandemic. there are ongoing researches to develop effective therapeutics and vaccines against COVID-19 using various methods, and many results have been published. The structure-based drug design of SARS-CoV-2 related proteins is promising. However, 
reliable information regarding the structural and intra- and intermolecular interactions is required. We have conducted studies based on the fragment molecular orbital (FMO) method for calculating the electronic structure of protein complexes and analyzing their quantitative molecular interactions. This enables us to extensively analyze the molecular interactions in residues or functional group units acting inside protein complexes. Such precise interaction data are available in the FMO database (FMODB) (https://drugdesign.riken.jp/FMODB/). Since April 2020, we have performed several FMO calculations on the structures of SARS$\mathrm{CoV}-2$ related proteins registered in the Protein Data Bank. We have published the results of 681 structures, including three structural proteins and eleven nonstructural proteins, on the COVID-19 special page (as of June 8, 2021). In this paper, we describe the entire COVID19 special page of FMODB and discuss the calculation results for various proteins. These data not only aid the interpretation of experimentally determined structures but also the understanding of protein functions, which is useful for rational drug design for COVID-19. 


\section{Introduction}

The COVID-19 pandemic has been ongoing since its declaration by WHO in March 2020, and as of June 8, 2021, it had killed 3,718,944 people and infected 172 million people worldwide ${ }^{1}$. COVID-19 has had a devastating impact on global health and economic activities, and the loss of social infrastructure due to urban lockdown has reached unprecedented levels, with no signs of abatement. To fight against COVID-19, there is a need to understand the causative virus, SARS-CoV-2, and develop effective vaccines and therapies. Consequently, structural analyses of SARS-CoV-2 related proteins have been rapidly conducted and are available worldwide in the Protein Data Bank $(\mathrm{PDB})^{2}$. In addition to experimental structures, researchers worldwide collate model structures, MD simulation results, and bioinformatics data and made them available as their data resources ${ }^{3}$. The PDB Japan (PDBj) ${ }^{4,5}$ has categorized PDB data of SARS-CoV-2 related proteins into "All entries," "Representative entries," and "Latest entries" on the COVID-19 special page, and 1,277 structures are available as of June 8, 2021. Viral proteins include four structural proteins (SP) that form virus particles and 16 nonstructural proteins (NSP) intracellularly produced after infecting human cells. The structures of three SP and thirteen NSP have already been clarified by cryoelectron microscopy (Cryo-EM), X-ray, and NMR. Though these structural data are very useful information for developing therapeutic agents, there is a need to precisely calculate and clarify how these viral proteins interact with each other and when candidate therapeutic compounds bind strongly to the proteins.

We have performed several fragment molecular orbital (FMO) ${ }^{6,7}$-based quantum chemical calculations on the entire structure of SARS-CoV-2 related proteins, focusing on the representative entries of $\mathrm{PDBj}$, and all the results have been published in the FMO database (FMODB) ${ }^{8} 9$ since April 2020. In FMODB, all FMO calculation results can be downloaded and molecular interaction analysis can be performed through the web interface and BioStation Viewer software ${ }^{10}$. In this paper, we describe FMO calculation results (681 structures as of June 8, 2021) available on the COVID-19 special page of FMODB.

\section{Method}

\subsection{Molecular Modeling}

We performed FMO calculations for 681 protein structures, mainly from the representative entries published on the COVID-19 special page of $\mathrm{PDBj}$, to reveal their precise interaction energies. $\mathrm{MOE}^{11}$ was employed for molecular modeling, and structural refinement was performed according to the resolution of the registered structures following the procedure shown in Figure 1. Here, missing atoms in the PDB structure are complemented, and appropriate structural optimization is performed. The level of structural optimization is 
changed according to the experimental method and its resolution, but for some data, exceptions are made, such as performing structural optimization for nonhydrogen atoms, even when the resolution is less than 2.0.

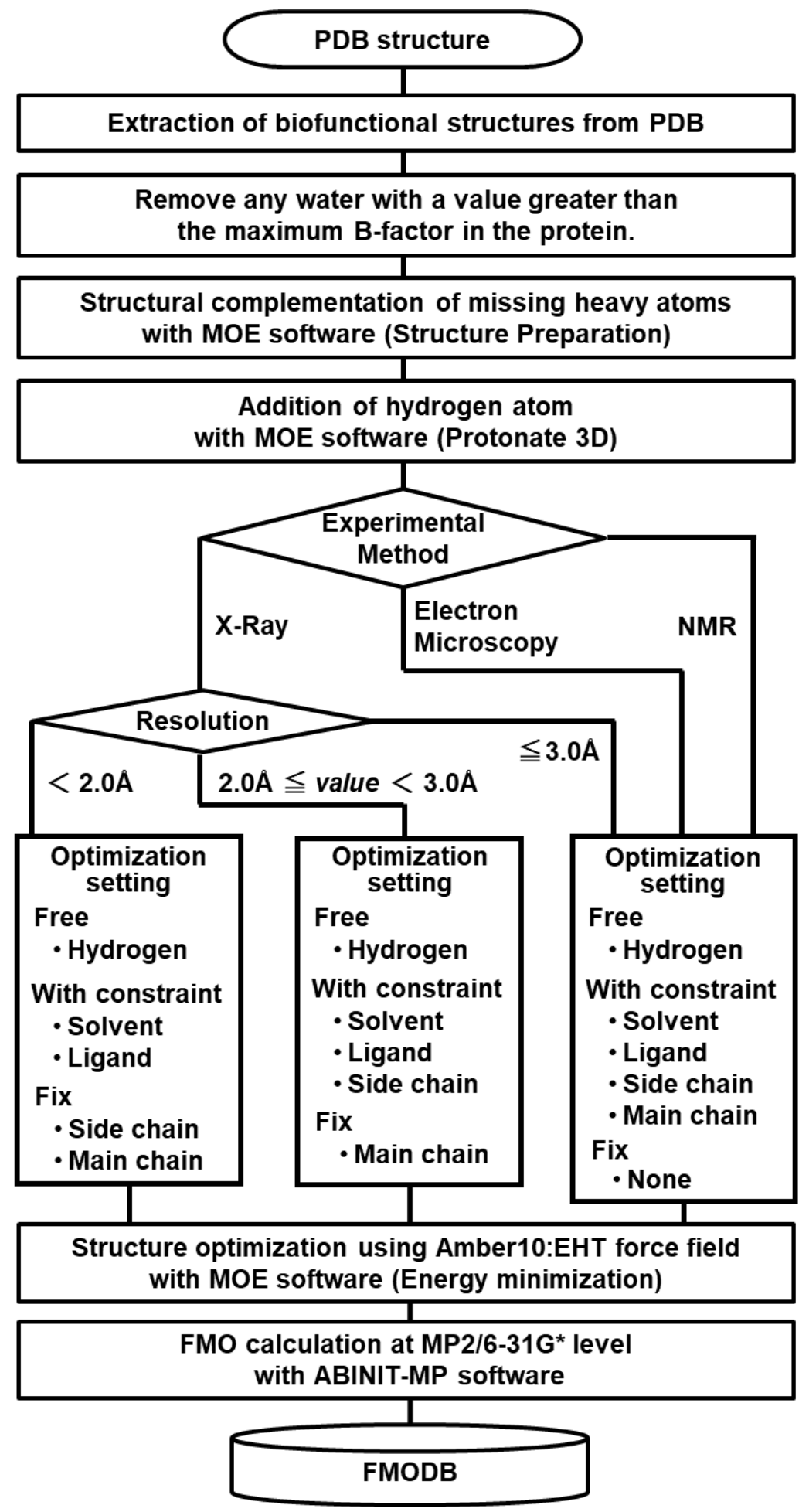


Figure 1. Sequence of workflow from downloading PDB structure to FMODB registration

\subsection{FMO Calculation}

The basic fragmentation method for FMO calculations is as follows: proteins are fragmented into amino acid residue units, nucleic acids are fragmented into backbone and base units, and ligands are fragmented into one or several fragments ${ }^{12}$. Quantum chemical calculations were performed at the theoretical level of FMO2-MP2/6-31G*13,14 to calculate the total energy $\left(E_{\text {total }}\right)$, atomic charge, and interfragment interaction energy (IFIE; $\left.\Delta \tilde{E}_{I J}\right)$.

$$
E_{\text {total }}=\sum_{I} E_{I}^{\prime}+\sum_{I>J} \Delta \tilde{E}_{I J}
$$

IFIE can be further decomposed into four energy components, including electrostatic (ES), exchange repulsion (EX), charge transfer with higher-order terms $(\mathrm{CT}+\mathrm{mix})$, and dispersion (DI) terms. This is known as the pair interaction energy decomposition analysis (PIEDA) ${ }^{15}$ ${ }^{16}$, and it is expressed as follows:

$$
\Delta \tilde{E}_{I J}=\Delta \tilde{E}_{I J}^{\mathrm{ES}}+\Delta \tilde{E}_{I J}^{\mathrm{EX}}+\Delta \tilde{E}_{I J}^{\mathrm{CT}+\mathrm{mix}}+\Delta \tilde{E}_{I J}^{\mathrm{DI}}
$$

PIEDA is vital in applying the FMO method to drug discovery because it gives information about the characteristics of the interaction, in addition to their magnitude (stable or unstable). For interpreting PIEDA, please refer to FMO books ${ }^{717}$ and the original reference ${ }^{15}$. Hydrogen bonding is mainly detected as the stabilization energy for the ES and CT + mix terms, and hydrophobic type of interactions, such as $\mathrm{CH} / \pi$ and $\pi-\pi$, are mainly detected as the stabilization energy for the DI term.

Among the data registered in FMODB, for entries for which the binding energy can be defined, such as protein-ligand, protein-protein, and protein-RNA, the binding energy is calculated from the sum of IFIEs. The binding energy between molecules $\mathrm{A}$ and $\mathrm{B}\left(\Delta \mathrm{E}_{\mathrm{AB}}\right)$ is expressed as follows:

$$
\Delta E_{A B}=\sum_{\substack{I \in A \\ J \in B}} \Delta \tilde{E}_{I J}
$$

All calculations in FMODB were performed using ABINIT-MP 18 19, a FMO calculation software. We used supercomputers, including TSUBAME3.0 at Tokyo Institute of Technology, Oakforest-PACS at JCAHPC, and FUGAKU and HOKUSAI at RIKEN. 


\subsection{FMODB Registered Information and Web Interface}

The FMO calculation results of COVID-19-related proteins are available from the top page of FMODB or the COVID-19 special page ${ }^{8}$. Each FMODBID page contains information on the used PDB structures and calculation conditions, and the entire dataset, including ABINIT-MP input and output files, can be downloaded. In addition, the binding energies and IFIE/PIEDA energy lists are provided to analyze the interaction data using the web interface. BioStation Viewer ${ }^{10}$ can be used to perform a detailed analysis using the downloaded data. As an example, Figure 2 shows a detailed view of FMODB for the complex structure of the main protease and ligand. The upper part shows the structural data and calculation conditions, and the lower part shows the interaction energies with amino acid residues near the ligand by the PIEDA energy component. The bottom chart shows the interaction energies with amino acid residues near the ligand by the PIEDA energy component. Conditions, such as the range to be displayed, can be specified.

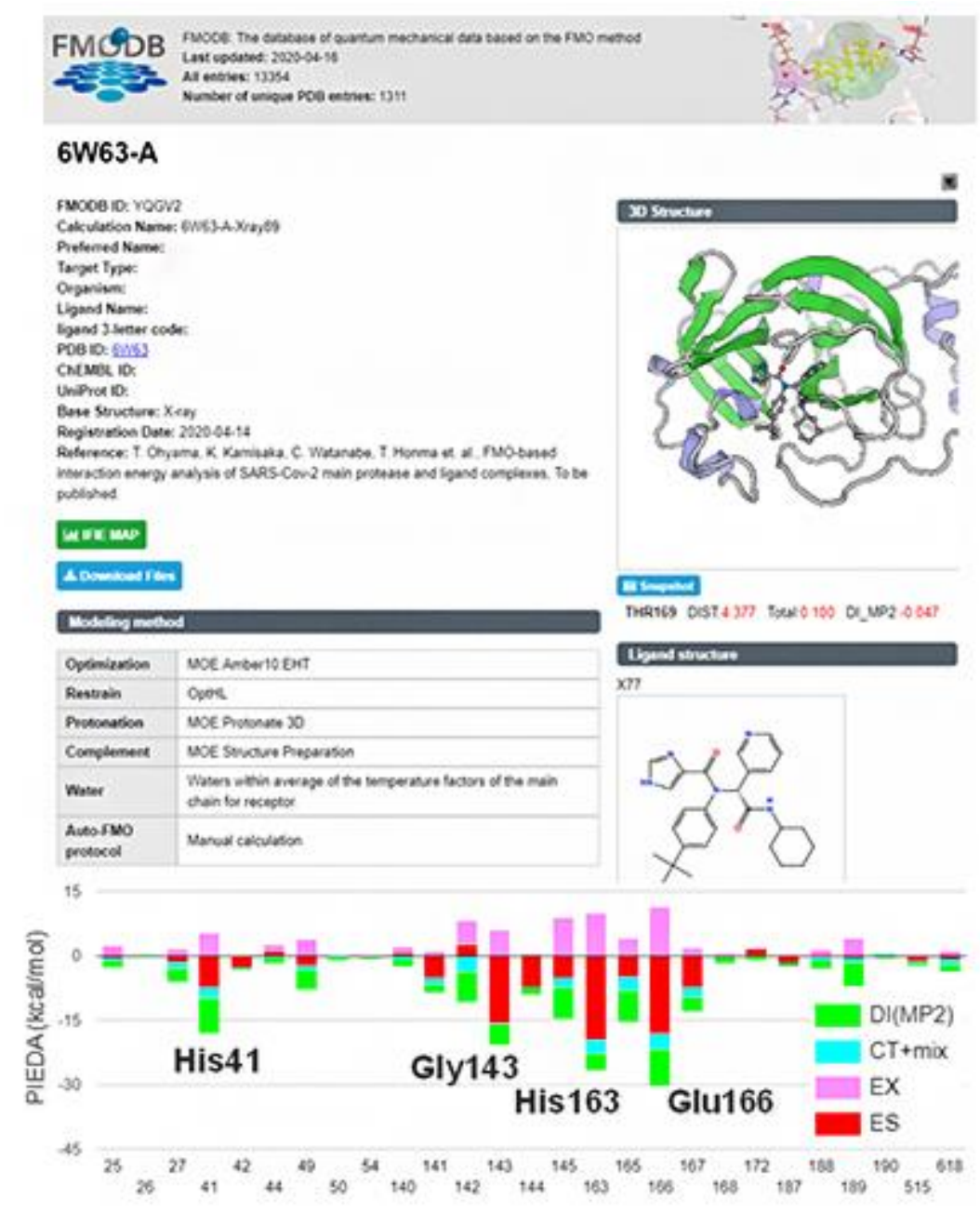




\section{Results and Discussions}

SARS-CoV-2 is a single-strand positive-sense RNA virus composed of four SPs and RNA genes. After infecting a host cell, the virus synthesizes 16 types of NSPs. Spike (S) protein, a type of SP that protrudes from the viral surface, plays an important role in the process of viral infection of the hosts, making it an important target for vaccines and therapeutic agents ${ }^{20,21}$. For NSPs, protease and RNA-dependent RNA polymerase (RdRp) are also promising drug targets. Two proteases of SARS-CoV-2 are involved in the cleavage of polyproteins synthesized from RNA to produce various proteins. The main protease (Mpro) cleaves polyprotein at eleven sites, and the papain-like protease (PLpro) cleaves polyprotein at three specific sites ${ }^{22}$. RdRp and Helicase are key enzymes in gene duplication by creating replication and transcription complex (RTC) ${ }^{23}$.

The number of registered structures at June 8, 2021 is shown in Figure 3. About 50\% of the 681 structures registered in FMODB are Mpro, followed by S protein and PLpro. In addition, ADP ribose phosphatase, Endoribonuclease, 2'-O-ribose methyltransferase, Helicase, etc., have been widely registered. The number of entries per protein depends on the number of representative entries in the PDBj site, and FMODB preferentially collects the structures with the highest resolution among the PDB entries with 100\% identical amino acid sequences, i.e., representative structures in PDBj. FMODB also preferentially collects the structures whose primary citation information is publicly available from the PDB site. 


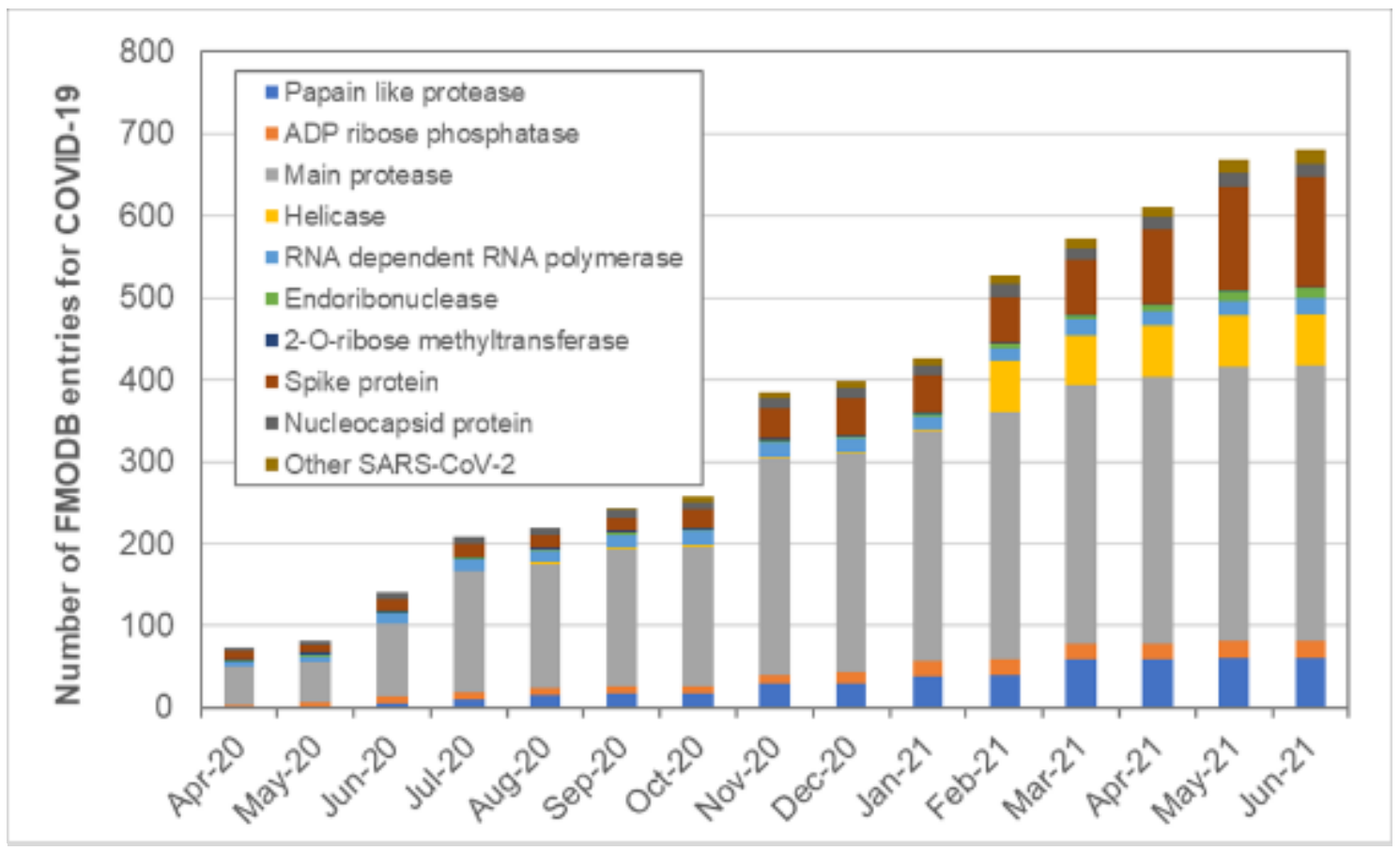

Figure 3. Number of registered structures in FMODB

The following is a typical example of registered structures.

\subsection{Papain-like protease (nsp3)}

PLpro is an essential coronavirus enzyme required for the cleavage of viral polyproteins at three sites, and the catalytic residues for processing are Cys111 and His272 24,25 . The substrate-binding site of PLpro is exposed to solvent and contains a flexible $\beta$-hairpin loop (G266-G271) called the blocking loop 2 (BL2), which changes its structure significantly depending on the substrate and inhibitor configuration ${ }^{26}$. PLpro is also involved in the cleavage of post-translational modifications of proteins on host proteins (deubiquitination) as an evasion mechanism against host antiviral immune responses ${ }^{27,} 28$. Here are some examples of covalent and noncovalent ligand complexes registered in FMODB.

As an example of FMO calculations, we show the results for the X-ray crystal structure of the tetrapeptide Ac-hTyr-Dap-Gly-Gly-VME (VIR251) complex, which is one of the peptide inhibitors of PLpro and is covalently bound to PLpro at Cys111 (PDB ID: 6WX429, FMODB ID: YQG52). VIR251 strongly inhibits the activity of both SARS-CoV PLpro and SARS-CoV$2 \mathrm{PLpro}^{29}$. Although VIR251 is a four-residue ligand, we treated the entire ligand as a single fragment (the residue names of the ligands are registered as LIG).

Figure 4 shows the interaction between the entire ligand molecule and its surroundings and the interaction energy quantified by PIEDA. The binding energy between VIR25 and SARS- 
CoV-2 PLpro, according to Equation (3), was $-170.6 \mathrm{kcal} / \mathrm{mol}$ (except for the interaction with Cys111, which is covalently bound to VIR251). ES ( $-132.9 \mathrm{kcal} / \mathrm{mol})$ contributed most to the binding energy, followed by DI $(-84.2 \mathrm{kcal} / \mathrm{mol})$ and CT $(-41.3 \mathrm{kcal} / \mathrm{mol})$. Since VIR251 has a positive charge $(+1 \mathrm{e})$, the ES interaction $(-107.2 \mathrm{kcal} / \mathrm{mol})$ with the acidic residue Asp164, which forms a hydrogen bond with the $\mathrm{NH}$ of hTyr, contributes significantly to the binding energy. In addition, the ES and CT components of hydrogen bond forming residues, such as Gly163, Tyr264, Tyr268, Gly271, etc., and the DI component due to $\mathrm{CH} / \pi$ interactions with surrounding hydrophobic residues, such as Leu162, Pro248, Tyr264, etc, also contributed significantly to the binding energy.

In the interaction with the catalytic His2 22 fragment, a dispersion interaction derived from the imidazole ring of His272 was observed, in addition to the hydrogen bond with the $\mathrm{C}=\mathrm{O}$ derived from Gly271. Note that the $\mathrm{C}=\mathrm{O}$ from Gly271 belongs to the His272 fragment due to fragmentation. Strong interactions between VIR251 and several water molecules were also detected, suggesting that the role of water molecules in bridging ligands and PLpro is important in designing new inhibitors. Details of these energetic analyses can be performed freely using FMODB.

(a)

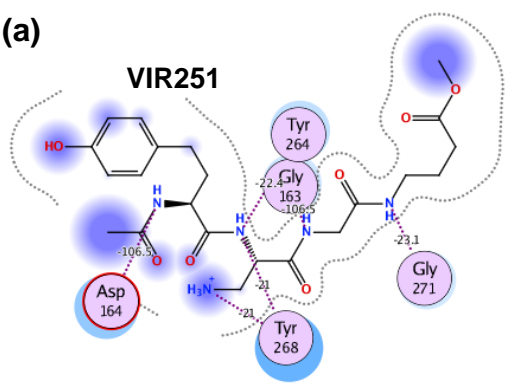

(b)
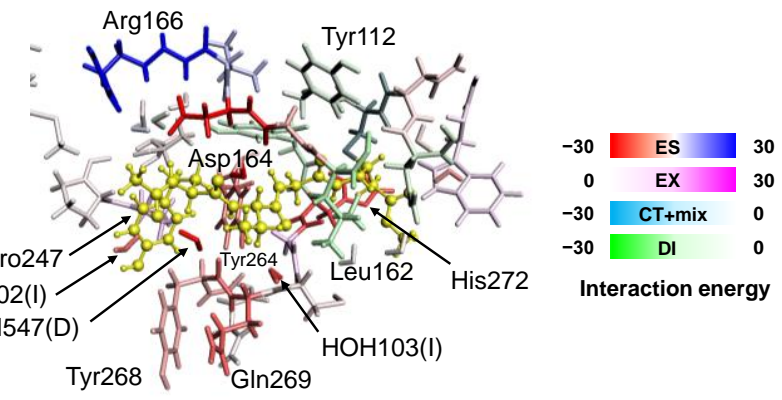

(c)

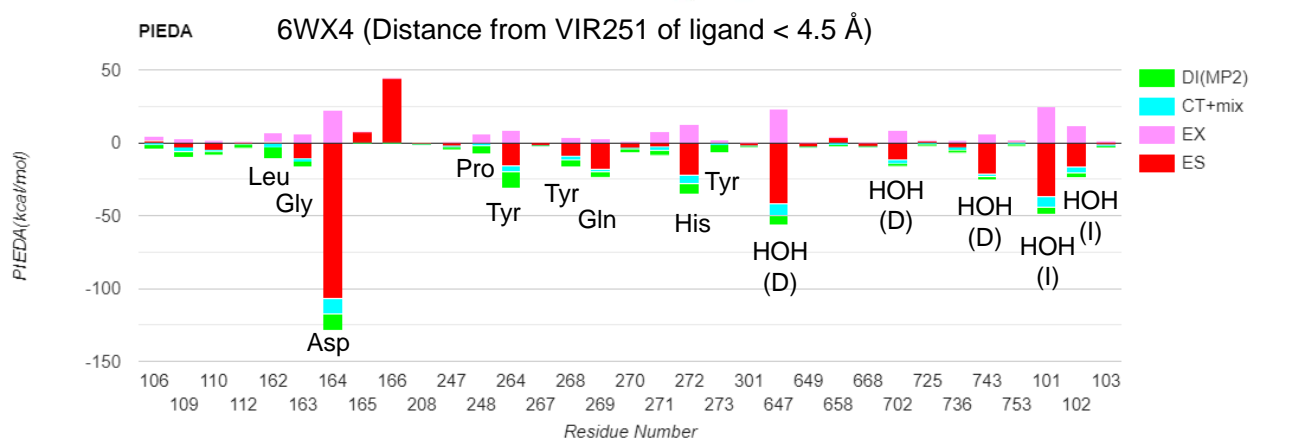

Figure 4. Interaction between papain-like protease (PLpro) and its peptide inhibitor, VIR251 (PDBID: 6WX4,

FMODB ID: YQG52). (a) The interaction site between the entire ligand molecule and the surrounding residues, as drawn by MOE. (b) PIEDA main component between the ligand (yellow) and a residue fragment within $4.5 \AA$, mapped on the molecular structure using a BioStation Viewer. (c) Graphical representation of the PIEDA components as in (b) using the FMODB web interface. Energy values are in 
$\mathrm{kcal} / \mathrm{mol}$.

Next, FMO results for the X-ray crystal structure of the tetramer with the neutral molecule GRL0617 bound as a noncovalent ligand have also been registered for each monomer (PDB ID: 7CMD ${ }^{26}$, FMODB ID: R1278, Z284N, 6321Z, 2KQVR). GRL0617 has an $\mathrm{IC}_{50}$ of $2.2 \pm$ $0.3 \mu \mathrm{M} / \mathrm{L}$ and is considered a promising candidate compound ${ }^{26}$. The average binding energy of GRL0617 and each monomer registered in FMODB was $-112.5 \pm 2.7 \mathrm{kcal} / \mathrm{mol}$, which is about two-thirds of that of the positively charged covalent ligand VIR251. The number of interacting residues was also smaller than that of VR251, but the $\mathrm{CH} /$ interaction with Tyr268 in the BL2 loop, the hydrogen bond with Gln269, and the hydrogen bond and $\mathrm{CH} /$ interaction with Asp164 in the $\alpha 3$-to- $\alpha 4$ loop were large (Figure 5), suggesting that GRL0617 inhibits the inversion of the BL2 loop by interacting with these loops, thereby inhibiting the recognition of the substrate, which is consistent with the structural considerations ${ }^{26}$.

(a)

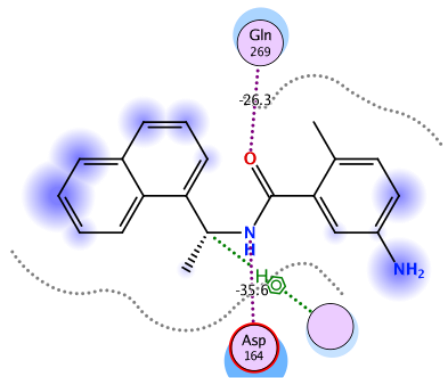

(b)

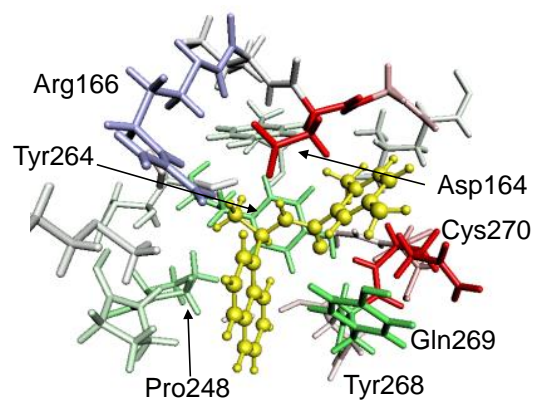

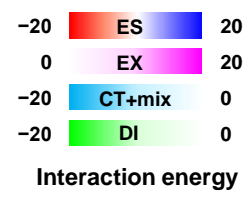

(c)

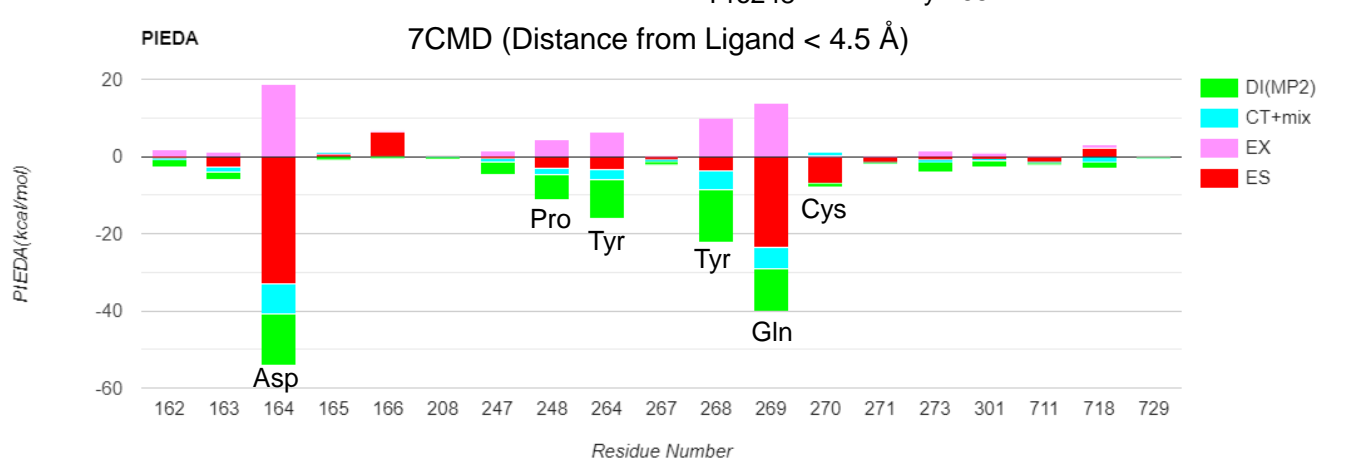

Figure 5. Interaction between PLpro and noncovalent ligand (PDBID: 7CMD-A). (a) The interaction site between the entire ligand molecule and the surrounding residues, as drawn by MOE. (b) PIEDA main component between the ligand (yellow) and a residue fragment within $4.5 \AA$, mapped on the molecular structure using a BioStation Viewer. (c) Graphical representation of the PIEDA components as in (b) using the FMODB web interface. Energy values are in $\mathrm{kcal} / \mathrm{mol}$. 


\subsection{ADP ribose phosphatase (nsp3)}

Nsp3 encodes the largest multidomain, including the ADP ribose phosphatase domain. Although its function has not yet been elucidated, it is inferred to be related to the ability of proteins to remove ADP-ribose from ADP-ribosylated proteins and $\mathrm{RNA}^{30}$. The $\mathrm{X}$-ray crystal structures of the complexes with ADP, AMP, etc. have been analyzed, and they include catalytic water ${ }^{30}$.

The complex of ADP ribose phosphatase and ADP ribose (PDBID: 6W02, FMODB ID: 1JLYZ, Figure 6a), which is drawn based on the atomic coordinates of the PDB crystal structure, shows that only the interaction between Asp22 and Lys44 is extracted. On the other hand, the PIEDA energies of the adenosine portion of ADP ribose in FMO calculations (Figures 6b,c) show that adenine is stabilized by DI with Phe156 and ES with Asp22 and Ile23 ( $\mathrm{C}=\mathrm{O}$ of the Asp22 main chain in the biochemical description). The side chains of Phe156 and Ile23 interact with the adenine base in a $\mathrm{CH} / \pi$ interaction, indicating a relatively large DI component. A similar analysis on the diphosphate moiety of the ligand showed strong ES interactions with the two surrounding hydrogen-bonded water molecules, Val49, Gly130, Ile131, and Phe132. Analysis of the ribose moiety showed strong ES interactions with Ala39, Asn40, and Gly48. Lys44 also showed strong ES interactions with diphosphate moiety, although they were separated by more than $4.5 \AA$. The details of the interaction energy values from these single fragments are available on the FMODB web interface. When the ligand consists of multiple fragments, the BioStation Viewer ${ }^{10}$ can be used for more detailed ligand interaction energy analysis. Figure S1 shows a mapping of the interaction energies between the entire ligand molecule and the surrounding residues. From the whole ligand molecule, the interactions with two water molecules (HOH332 and HOH384) and Gly130, which are involved in the phosphatase activity, are more clearly shown. 
(a)

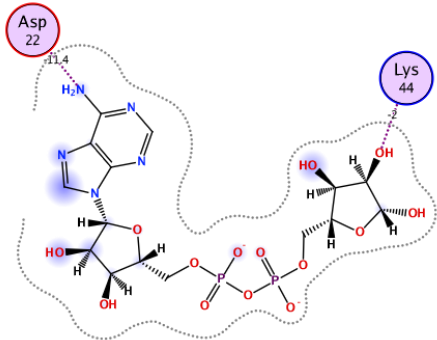

(b)

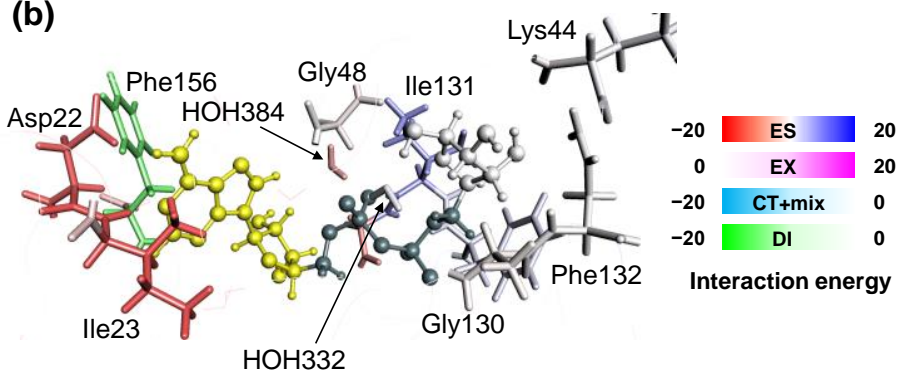

(c)

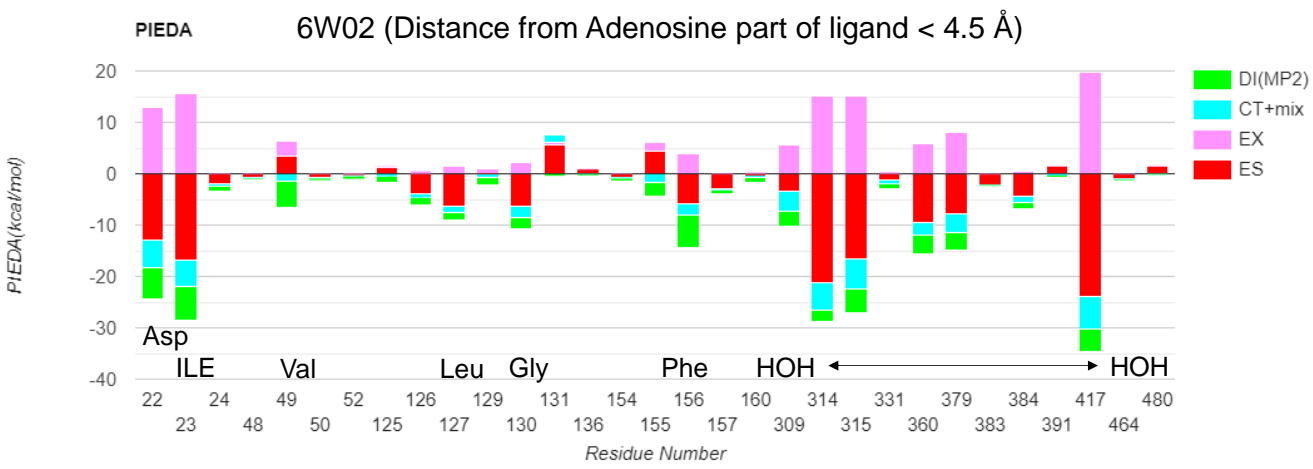

Figure 6. Interaction between ADP ribose phosphatase and ADP ribose as a ligand (PDBID: 6W02,

FMODB ID: 1JLYZ). (a) The interaction site between the entire ligand molecule and the surrounding residues, as drawn by MOE. (b) PIEDA main component between the adenosine portion of the ligand (yellow) and a residue fragment within $4.5 \AA$, mapped on the molecular structure using a BioStation Viewer. (c) Graphical representation of the PIEDA components as in (b) using the FMODB web interface. Energy values are in $\mathrm{kcal} / \mathrm{mol}$.

\subsection{Main Protease (nsp5)}

Mpro is an enzymatic protein encoded in the viral genome, which is one of the two coronavirus proteases responsible for the cleavage of polyproteins translated from genomic RNA, and is involved in the maturation of viral proteins ${ }^{22}$. Mpro has been studied worldwide as one of the most important drug targets for COVID-19. In structural biology research, the first crystal structure of SARS-Cov-2 related protein was the covalent complex structure of Mpro and N3 inhibitor (PDBID: 6LU7 ${ }^{31}$ ). The structures of Mpro in PDB are obtained from $\mathrm{X}$-ray crystallography. Most of the structures are complex with ligands, and the binding mode is either noncovalent or covalent with Cys145. For the complex of Mpro and N3 inhibitor (PDBID: 6LU7), FMO calculations were performed and registered in FMODB by Hatada et al. ${ }^{32}$ when the PDB structure was published (FMODBID: R1GK8). In addition, statistical interaction analyses using FMO calculations for several sampled structures from classical MD calculations have been reported ${ }^{33}$. Among the COVID-19-related proteins in FMODB, Mpro has the highest number of registered structures. 
Mpro is a three-domain cysteine protease, and the substrate-binding pocket consists of four subsites (S1', S1, S2, and S4) and the Cys145-His41 catalytic dyad ${ }^{34,35}$. Here, we classify the features of the pockets regarding the interaction of 110 ligand complex structures registered in FMODB (Figure 7). Figure $7 \mathrm{a}$ shows the superposition of eight covalent and one noncovalent structures, which are classified as representative structures in PDBj. The covalent structures in particular contact all four subsites. We performed SOM-clustering analysis $^{36}$ of the interactions based on PIEDA energies for the 110 ligand complex structures. Figure $7 \mathrm{~b}$ shows 12 clusters to which three or more ligands belong. Most of the clusters have ligands localized to a single site: five clusters at the S1' site, two clusters at the S1 site, and one cluster at the S2 site. Four ligand clusters were bound across multiple sites.

(a)

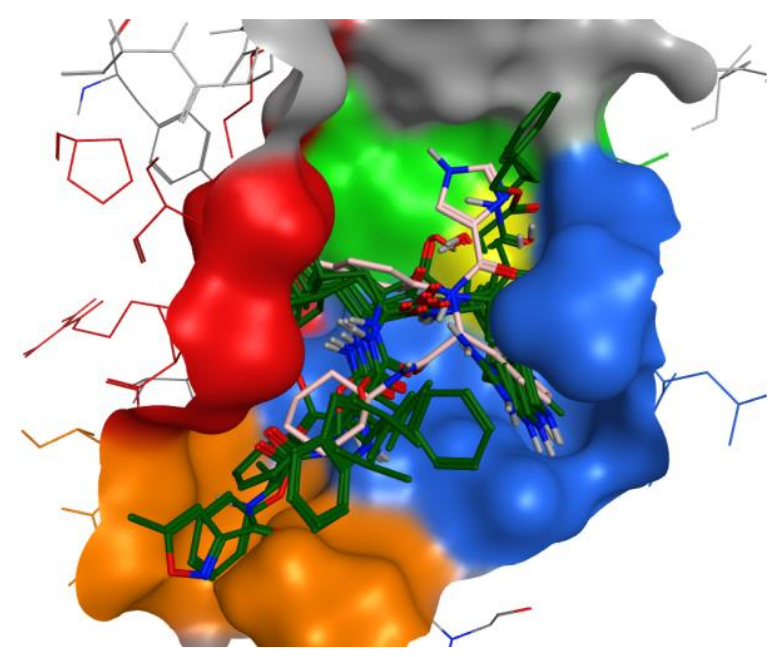

(b)

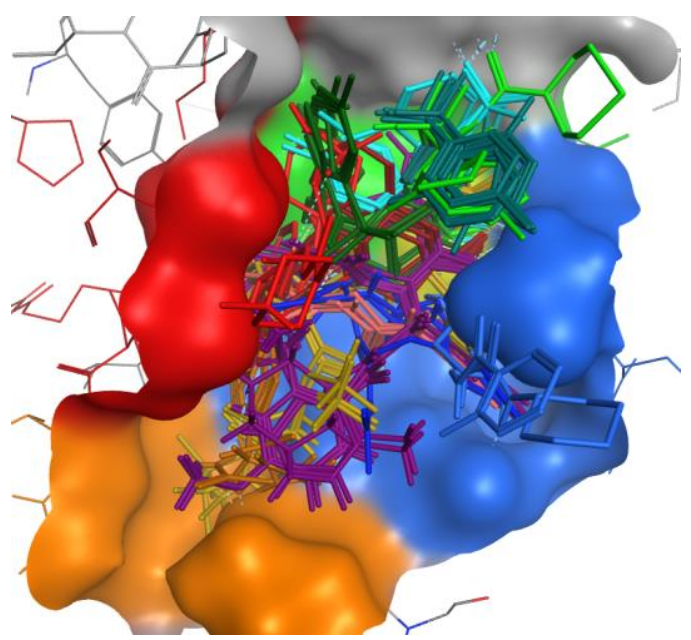

Figure 7. Substrate-binding pocket of main protease (Yellow area: Cys145; green area: S'1 subsite; blue area: S1 subsite; red area: S2 subsite,; orange area: S4 subsite). (a) Only ligands of complexes classified as representative structures in $\mathrm{PDBj}$ are indicated by sticks (green: 8 covalent ligands; pink: 1 noncovalent ligand). (b) Clustering of 110 complex structures and 12 types of clusters to which three or more ligands belong.

All FMO calculation results for these structures are registered in FMODB, and they can be accessed by anyone. Here, we discuss one example each of the covalent and noncovalent ligands shown Figure 7a. Figure 8 depicts the interaction of an example of covalent ligands (PDBID: 6WTT ${ }^{37}$, FMODBID: 4NVVN). The prodrug of peptide ligands, GC-376, is covalently bound to Cys145 of Mpro after changing to GC-373. The S1, S2, and S4 binding sites interact with the substituents of the ligand (Figure 8a), and PIEDA analysis showed that 
the interacting residues are His41, His163, Met165 (His164 C=O), Glu166, and Gln189 (Figure $8 \mathrm{~b}, \mathrm{c}$ ). In the S1 site, $\chi$-lactam of the ligand is hydrogen-bonded to His163, the side chain of Glu166, and Met165 ( $\mathrm{C}=\mathrm{O}$ of the His164 main chain in the biochemical notation), resulting in a strong ES + CT interaction. In the S2 site, the isopropyl group of the ligand interacts with His41 of the dyad in $\mathrm{CH} / \pi$. In the $\mathrm{S} 4$ site, the amide moiety of the ligand is hydrogen-bonded to the main chain of Glu166 and Gln189, which is stabilized by ES + CT. Gln189 also has a large DI component, which may be attributed to the dispersion interaction between the benzene ring of the ligand and the $\alpha$ and $\beta$ carbons of the Gln 189 side chain. The benzene ring also has a dispersion interaction with the methyl group of Met165 (Figure 8c) ${ }^{37}$. The binding energy is $-130.6 \mathrm{kcal} / \mathrm{mol}$, except for Cys 145 , which is covalently bound.

(a)

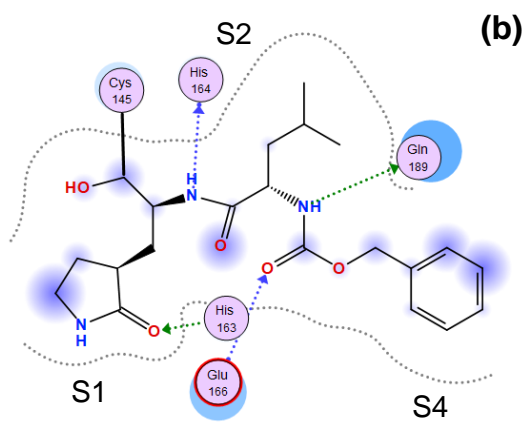

(b)

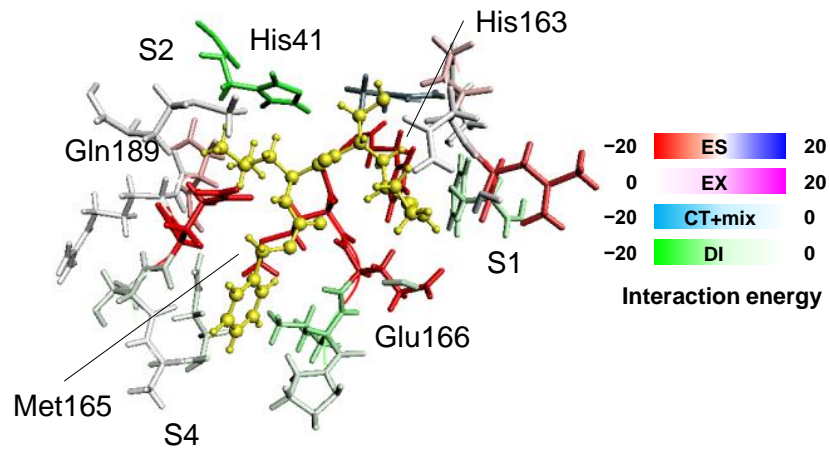

6WTT (Distance from Ligand $<4.5$ )

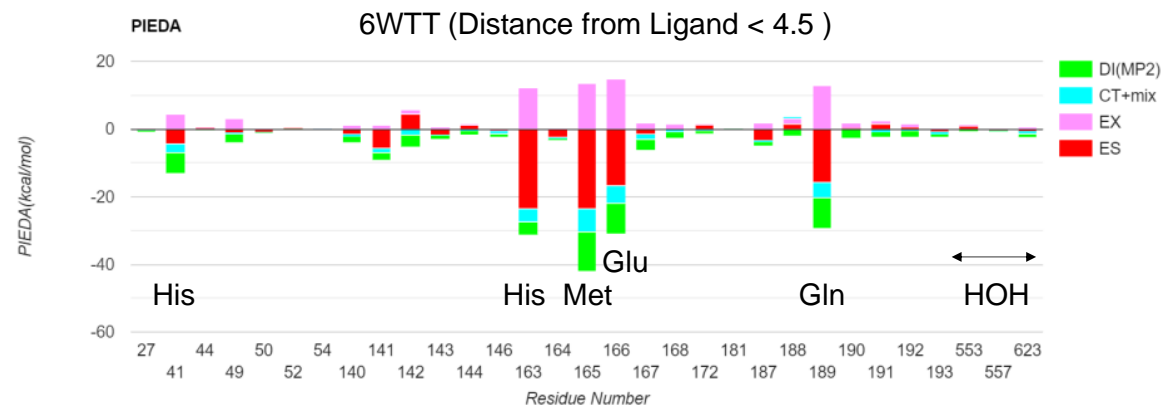

Figure 8. Interaction between main protease and covalently bound ligand (PDBID: 6WTT, FMODBID: 4NVVN). (a) The interaction site between the entire ligand molecule and the surrounding residues, as drawn by MOE. (b) PIEDA main component between the ligand (yellow) and a residue fragment within $4.5 \AA$, mapped on the molecular structure using a BioStation Viewer. (c) Graphical representation of the PIEDA components as in (b) using the FMODB web interface. Energy values are in kcal/mol.

In the X-ray structure of the complex of Mpro with the noncovalent ligand X77 (PDB ID: 6W79, FMODB ID: N3QNQ), the specific interactions with His41, Asn142, Gly143, His163, Met165, and Glu166 were confirmed by FMO calculations (Figure 9). According to the PIEDA component shown in Figure $9 b$ and $9 c$, as in the case of the covalent ligands, the $\mathrm{CH} / \pi$ 
interaction (DI) of His41 and the hydrogen bonds (ES + CT) between His163 and Met165 ( $\mathrm{C}=\mathrm{O}$ of His164) are maintained, but there is no DI in the Met165 side chain. For Glu166, the hydrogen bond in the main chain $(\mathrm{ES}+\mathrm{CT})$ is preserved, but the side chain hydrogen bond is absent, and $\mathrm{CH} / \pi$ interaction is formed between the $\beta$-carbon and the pyridine ring of the ligand (shown as the DI component). On the other hand, several additional interacting residues are observed around the imidazole ring. In particular, there is a $\mathrm{CH} / \mathrm{O}$ bond with Asn142 and a hydrogen bond with Glu143, as well as a dispersion interaction with Cys145 since it is a noncovalent ligand. The ligand binding energy is $-149.4 \mathrm{kcal} / \mathrm{mol}$, which is more stabilizing than that of the covalent ligand. Comparison between Figures $8 \mathrm{c}$ and $9 \mathrm{c}$ shows that the noncovalent ligand has more interactions than the covalent ligand.

(a)

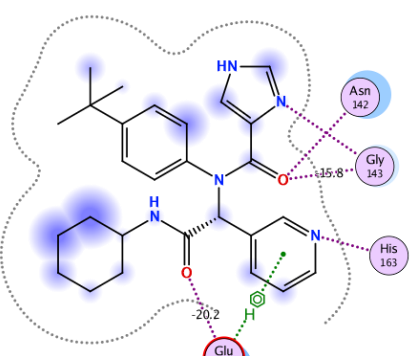

(b)

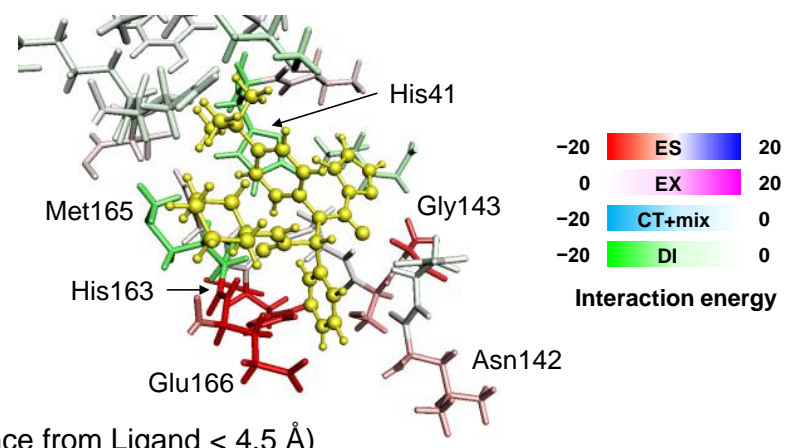

(c)

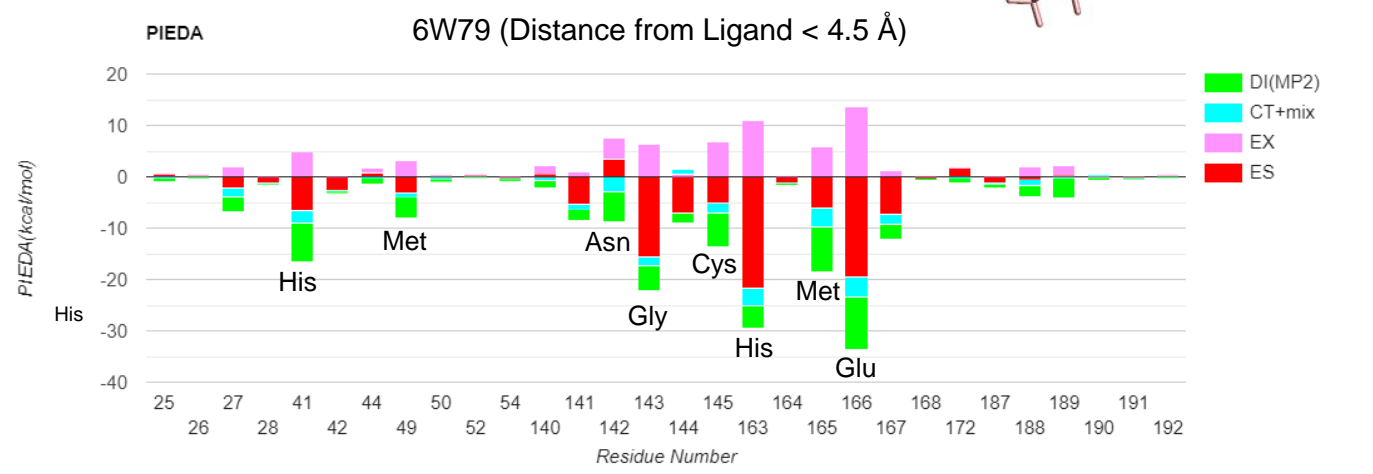

Figure 9. Interaction between main protease and noncovalent ligand (PDBID: 6W79-A, FMODBID:

N3QNQ). (a) The interaction site between the entire ligand molecule and the surrounding residues, as drawn by MOE. (b) PIEDA main component between the ligand (yellow) and a residue fragment within $4.5 \AA$, mapped on the molecular structure using a BioStation Viewer. (c) Graphical representation of the PIEDA components as in (b) using the FMODB web interface. Energy values are in $\mathrm{kcal} / \mathrm{mol}$.

\subsection{RNA-dependent RNA polymerase (nsp12) complex}


RdRp is an RNA synthesis enzyme involved in viral genome replication, forming RTC with cofactors nsp7,8,9 and helicase nsp13 $3^{23,} 38$ (Figure 10). RdRp is responsible for the replicating the genome, which is most important for the survival of the virus. RdRp is an important target of antiviral drugs, including remdesivir and favipiravir, and several ligand-containing PDB structures have been published. The structures registered in FMODB are RdRp-nsp7-nsp8-RNAremdesivir complex (PDBID: 7BV ${ }^{39}$, FMODBID: 1JL3Z ${ }^{40}$ ), complex without ligand (PDBID: 6YYT) ${ }^{38}$, complex without RNA (PDBIDs: 7BV1, 6M71, 7BW4), nsp7-nsp8

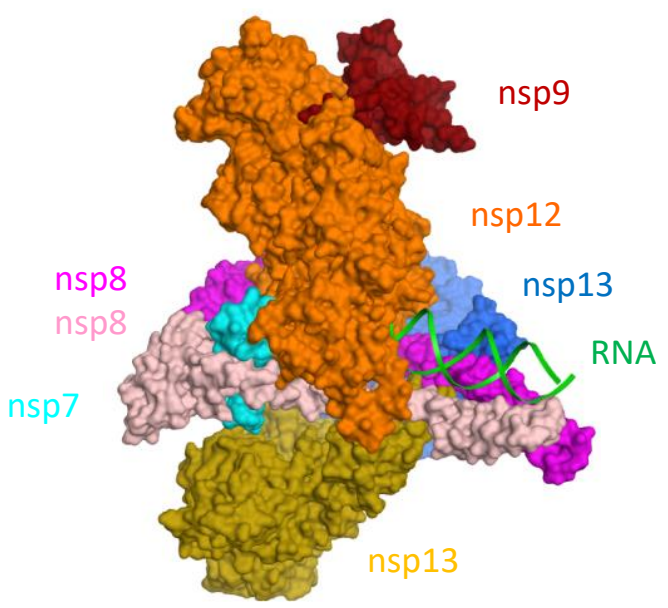

Figure 10. Replication and Transcription complex of SARS-CoV-2 8PDBID: 7CYQ) complexes, and nsp9 alone.

FMO calculations by Kato et al..$^{40}$ for the structure of the complex containing nucleic acid analog remdesivir show that remdesivir incorporated into the end of the template-primerRNA duplex retains nucleotide mimic interaction and is stabilized by hydrogen bonding between base pairs and $\pi-\pi$ stacking between inter- and intra-RNA strands (Figure 11). In particular, the stacking interaction is enhanced by the carbon-to-nitrogen substitution introduced into the adenine backbone of remdesivir. In addition, the interaction between remdesivir and RdRp is stabilized by hydrogen bonding with Asn691 and ES interaction with Asp760. Furthermore, the interaction of remdesivir with Thr687 is stabilized by $\mathrm{OH} / \pi$ interactions using $\pi$ electrons of the cyano group introduced into the sugar of remdesivir, in addition to hydrogen bonding. Thus, the interaction of remdesivir in the inhibition process of RNA strand elongation is clarified. In the interaction between the RNA duplex and the protein, $\operatorname{RdRp}$ (nsp12) is mainly responsible for stabilization (about $-600 \mathrm{kcal} / \mathrm{mol}$ ), nsp8 is responsible for about half $(-280 \mathrm{kcal} / \mathrm{mol})$, and nsp7 is responsible for $-24 \mathrm{kcal} / \mathrm{mol}$. Figure S2 shows a comprehensive interaction-energy diagram, IFIE-MAP. 
(a)

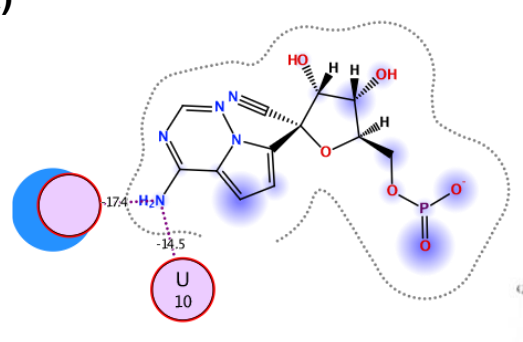

(b)

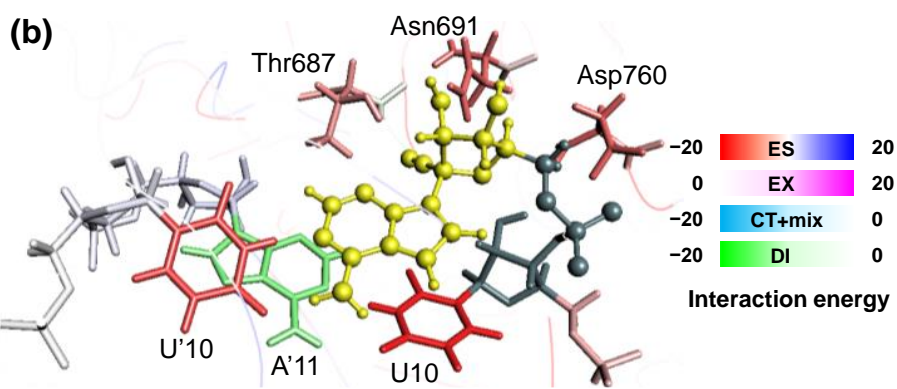

(c)

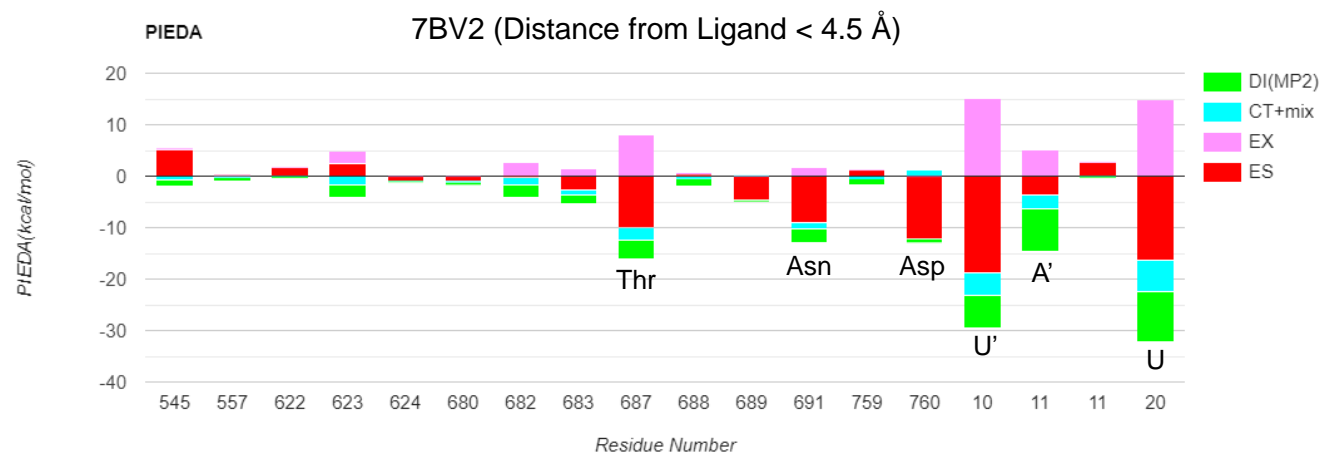

Figure 11. FMO results for RNA-dependent polymerase, nsp7, nsp8, RNA, and Remdesivir complex (PDBID: 7BV2, FMODBID: 1JL3Z). (a) The interaction site between the ligand (remdesivir) and the surrounding residues, as drawn by MOE. (b) PIEDA main component between the ligand (yellow) and a residue fragment within $4.5 \AA$, mapped on the molecular structure using a BioStation Viewer. (c) Graphical representation of the PIEDA components as in (b) using the FMODB web interface. Energy values are in $\mathrm{kcal} / \mathrm{mol}$.

\subsection{Helicase (nsp13)}

Helicase forms an RTC complex with RdRp, and both RdRp and helicase are essential for viral replication ${ }^{41}$. Helicase may be useful for smooth replication of the viral genome, such as backtracking $\mathrm{RdRp}^{42}$ and unwinding $\mathrm{RNA}^{43}$. FMODB has entries on the complex structure with (3-fluoro-4-methylphenyl) methanesulfonamide (PDBID: 5RL6, FMODBID: N3YLQ) and about 60 apo structures. The results of the complexes (Figure 12) show strong hydrogen bonding between the amide group of the ligand and Lys192, Tyr224, Thr228, and HOH833. Most of the interactions with the ligand are ES, but only Val226 exhibits $\mathrm{CH} / \pi$ interaction with the aromatic ring of the ligand, indicating stabilization of the DI component. However, in this calculation, the $\mathrm{Zn}$ atom coordinating to Helicase is neglected, and the FMO calculation that includes $\mathrm{Zn}$ is in progress. We shall also perform FMO calculations for RTC giant complexes (PDBID: 6XEZ, 7CXN, etc.) consisting of RdRp-helicase-RNA-ADP. 
(a)

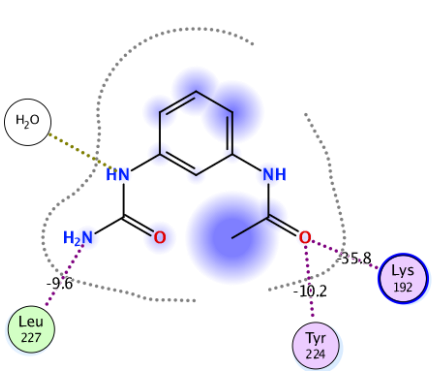

(b)

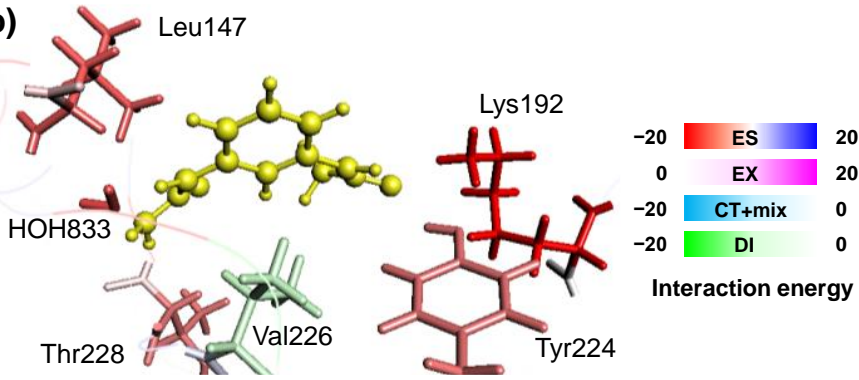

(c)

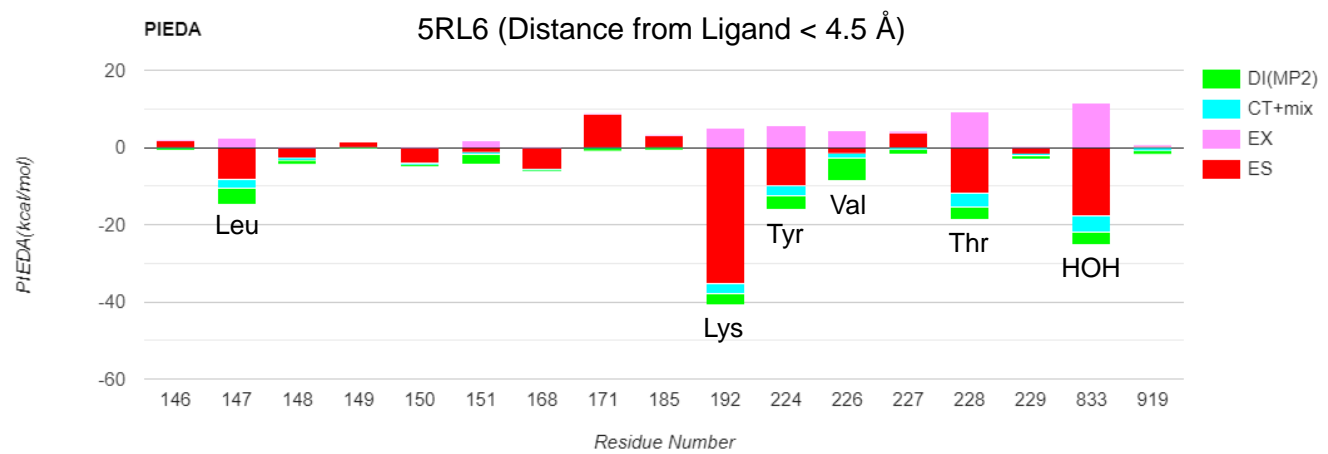

Figure 12. FMO results for Helicase-ligand complex (PDBID: 5RL6, FMODBID: N3YLQ). (a) The interaction site between the entire ligand molecule and the surrounding residues, as drawn by MOE. (b) PIEDA main component between the ligand (yellow) and a residue fragment within $4.5 \AA$, mapped on the molecular structure using a BioStation Viewer. (c) Graphical representation of the PIEDA components as in (b) using the FMODB web interface. Energy values are in $\mathrm{kcal} / \mathrm{mol}$.

\subsection{Endoribonuclease (nsp15)}

Endoribonuclease is a uridylate-specific endoribonuclease that cleaves the poly-U sequence of $\mathrm{RNA}^{44}$, and a structure with Uridine-5'-Monophosphate bound is available in PDB (PDBID: 6WLC, FMODB ID: N3G7Q) ${ }^{45}$. The coordinate information of the crystal structure (Figure 13a) suggests only Ser294 as an interacting residue between the whole ligand molecule and its surroundings. On the other hand, the PIEDA interaction energies calculated by FMO (Figure 13b,c) show a strong ES interaction between the uridine portion of the ligand and Lys290 and Ser294, as well as stabilization by DI interactions with Tyr343. A similar analysis on the monophosphate moiety of the ligand showed strong ES interactions with hydrogen-bonded Tyr343, water molecules (HOH540, HOH646), and Lys290. Figure S3 shows the interaction energies of the entire ligand molecule and its surroundings mapped onto the structure. From the whole ligand molecule, strong ES interactions with Ser294 and Tyr343, which are thought to be important for RNA recognition and degradation, and stabilization by DI interactions with His250 are observed. 
(a)

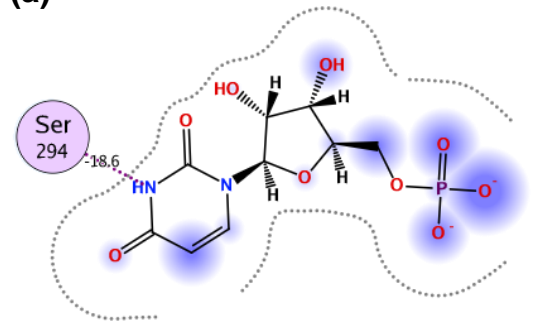

(b)

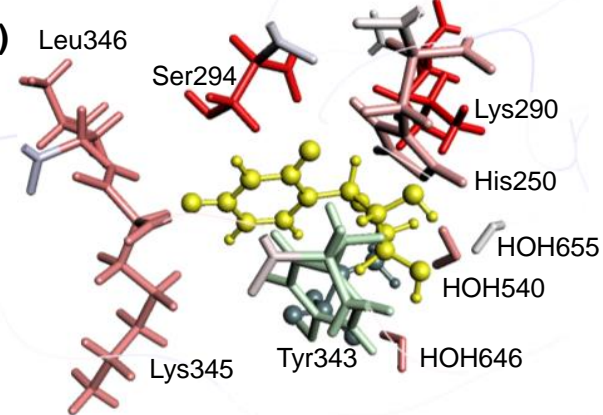

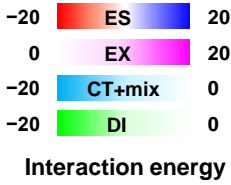

(c) PIEDA $\quad 6$ WLC (Distance from Uridine part of the ligand $<4.5 \AA$ )

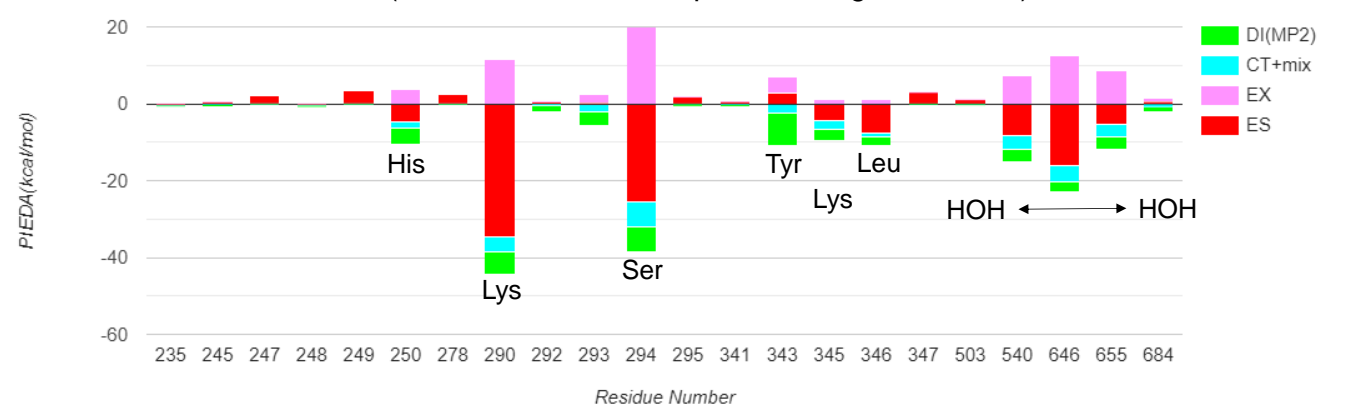

Figure 13. FMO results for Endoribonuclease -ligand complex (PDBID: 6WLC, FMODB ID: N3G7Q). (a) The interaction site between the entire ligand molecule and the surrounding residues, as drawn by MOE.

(b) PIEDA main component between the uridine portion of the ligand (yellow) and a residue fragment within $4.5 \AA$, mapped on the molecular structure using a BioStation Viewer. (c) Graphical representation of the PIEDA components as in (b) using the FMODB web interface. Energy values are in $\mathrm{kcal} / \mathrm{mol}$.

\subsection{2'-O-ribose Methyltransferase (nsp16)}

Nsp16 is a methyltransferase that forms a complex with nsp10 to transfer a methyl group from a donor to an acceptor, catalyzing methylation at the ribose $2 ' \mathrm{O}$ position of nucleotides at the end of viral RNA. The crystal structures of nsp16, nsp10, and their complexes with the methyl group donor, S-adenosylmethionine (SAM), or demethylation product, S-adenosyl-Lhomocysteine $(\mathrm{SAH})$, have been published ${ }^{46,}{ }^{47}$. The results of the analysis of the SAM complex are shown in Figure 14 (PDBID: 6W4H ${ }^{47}$, FMODBID: JM9Y9), The coordinate information of the crystal structure (Figure 14a) suggests an interaction of three Asps (6897, 6912, 6928) with Gly6869 and Tyr6845. On the other hand, PIEDA results show that the positively charged SAM is stabilized by strong ES interactions with Asp6897, Asp6912, Asp6928, Asp6931, Asn6841, and Ala6870 (Figures 14b,c). The binding pocket of SAM is composed of amino acids N43 (Asn6841), Y47 (Tyr6845), G71 (Gly6869), G81 (Gly6879), D99 (Asp6897), N101 (Asn6899), C115 (Cys6913), and D130 (Asp6928), all of which are 
conserved in SARS-CoV and MERS-CoV ${ }^{46}$. The corresponding residues in Figure $14 \mathrm{c}$ are underlined. FMO analyses showed that the largest interactions are in the order of D99 (Asp6897), which is hydrogen-bonded to the hydroxyl group of the adenosine sugar backbone of SAM, D130 (Asp6928), and N43 (Asn6841), which are hydrogen-bonded to the amine and carboxyl groups of the methionine portion of SAM, respectively. Recently, a complex structure of methylated CAP-like RNA and SAH (PDBID: 7LW3) was published, and FMO calculations are ongoing.

(a)

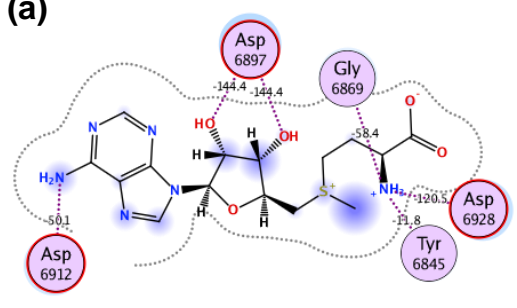

(b)

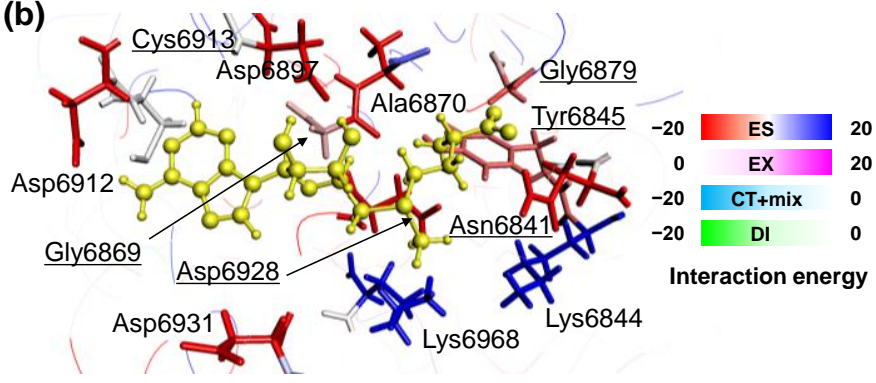

(c)

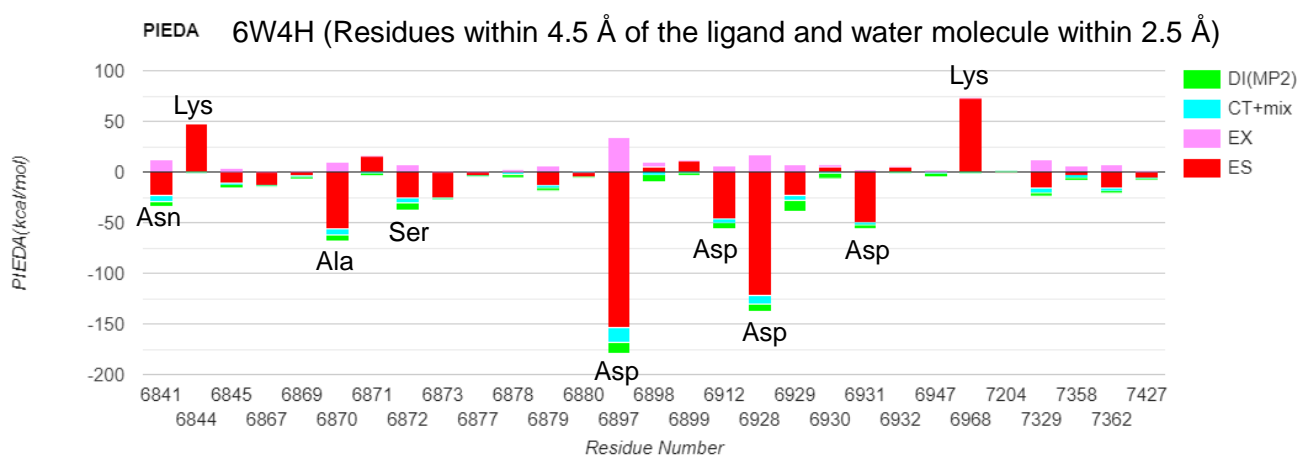

Figure 14. FMO results for 2'-O-ribose Methyltransferase-SAM complex (PDBID: 6W4H, FMODB ID: JM9Y9). (a) The interaction site between the entire ligand (SAM) and the surrounding residues, as drawn by MOE. (b) PIEDA main component between the ligand (yellow) and a residue fragment within $4.5 \AA$ and water molecure within $2.5 \AA$, mapped on the molecular structure using a BioStation Viewer. (c) Graphical representation of the PIEDA components as in (b) using the FMODB web interface. Energy values are in $\mathrm{kcal} / \mathrm{mol}$.

\subsection{Spike (S) protein}

One of the SP encoded in the viral genome, spike (S) glycoprotein, is a characteristic feature of coronaviruses, protrusions on the surface of the viral particle that binds to the ACE2 receptor on the host cell and allows the virus to enter the cell20, 21 . S protein has the highest number of published PDB entries, along with Mpro. Cryo-EM is the main tool for the structural analysis of the whole trimer, and X-ray data are also available for the receptor- 
binding domain (RBD)-antibody substructure. Since FMO calculations for the whole trimer are large-scale that cannot be done easily, most of them have been done for RBD and complexes with antibodies or ACE2 receptors. The champion data of open/close type structure of S protein trimer (PDBID: 6VXX, 6VYB) by Akisawa et al. using FUGAKU supercomputer ${ }^{48}$ are under preparation for registration to FMODB. For RBD substructures, C. Watanabe et al. have analyzed complexes with ACE2 and compared them with SARS ${ }^{49}$, and $\mathrm{K}$. Watanabe et al. have studied complexes with antibodies, including epitope analysis ${ }^{50}$ and mutant strains ${ }^{51}$, and all these data have been registered in FMODB. Here, we quantify the protein-protein interaction (PPI) of RBD-ACE2 and RBD-neutralizing antibodies and analyzed the interaction around the mutant residues Lys417, Asn501 in South African and Brazilian mutants reported since December $2020^{52,53,54}$ as examples of residue analyses.

An example of FMO-based PPI analyses using RBD of S protein and ACE2 complex (PDB ID: 6LZG, FMODB ID: 4NZVN) is shown in Figure 15. To identify the amino acid residues important in molecular recognition, we first mapped the main PIEDA components of the interactions between the whole ACE2 and each residue of the $\mathrm{S}$ protein onto the molecular structure of the $S$ protein (Figure 15a) and vice versa (Figure 15b). The strength of the interaction energy is indicated by a color gradation for each amino-acid residue. Since ACE2 is a -26 e charged protein, the charged amino-acid residues throughout the $\mathrm{S}$ protein interact electrostatically (Figure 15a). On the other hand, the RBD of S protein has a charge of $+2 e$, indicating that the interacting amino-acid residues are more concentrated at the binding interface compared to that in ACE2 (Figure 15b). Due to the large negative charge of ACE2, hydrogen bonds and $\mathrm{XH} / \pi$ interactions, which are key interactions in molecular recognition of $\mathrm{S}$ protein, are hidden by ES interactions of the charged residues. However, the hydrogen bond can be easily interpreted by analyzing the ES and CT terms of PIEDA, and the XH/ $\pi$ interaction can be easily interpreted by analyzing the DI term ${ }^{49}$. In addition, we performed PIEDA analysis of the mutant residues of S protein, Lys417 and Asn501, in the South African and Brazilian mutants and the surrounding residues (Figures 15 b,c). Lys417 forms a salt bridge with Asp30 of ACE2 and shows the strongest attractive interaction $(-116.3 \mathrm{kcal} / \mathrm{mol})$ between the two proteins. On the other hand, the main contribution of Asn501 is ES with surrounding amino acid residues (Tyr41, Lys353, Gly354, etc.). In a previous study, it was reported that the N501Y mutation enhances the ability of S protein to bind to ACE2 by acquiring more hydrogen bonds and $\mathrm{XH} / \pi$ interactions than $\mathrm{WT}^{49}$. 
(a)

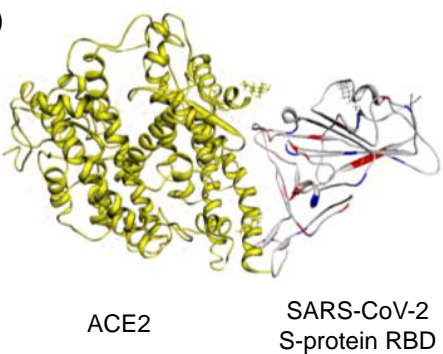

(b)

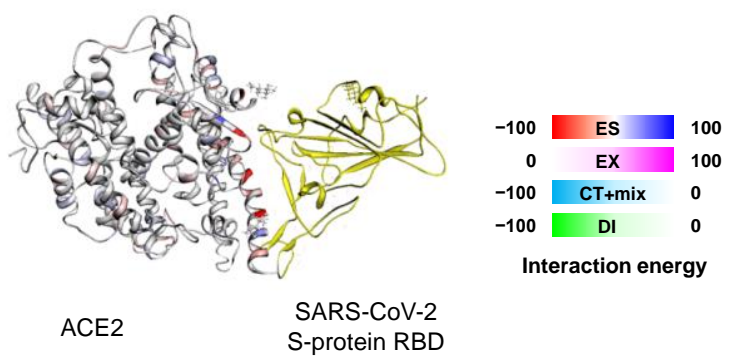

(c) PIEDA 6 LZG (Distance from fragment $509(417:$ Lys) $<4.5 \AA$ )

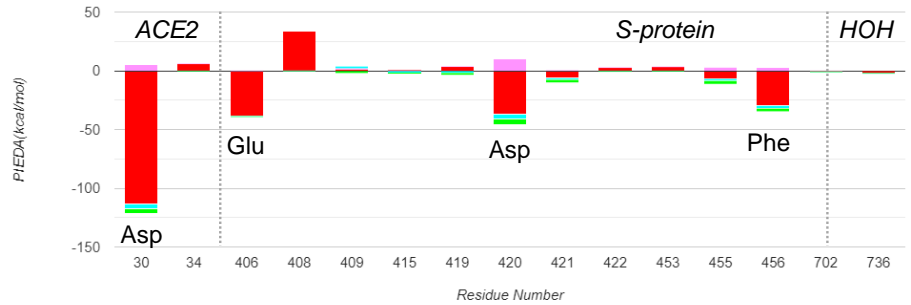

(d)

(e) PIEDA $6 L Z G$ (Distance from fragment $762(501:$ Asn $)<4.5 \AA)$
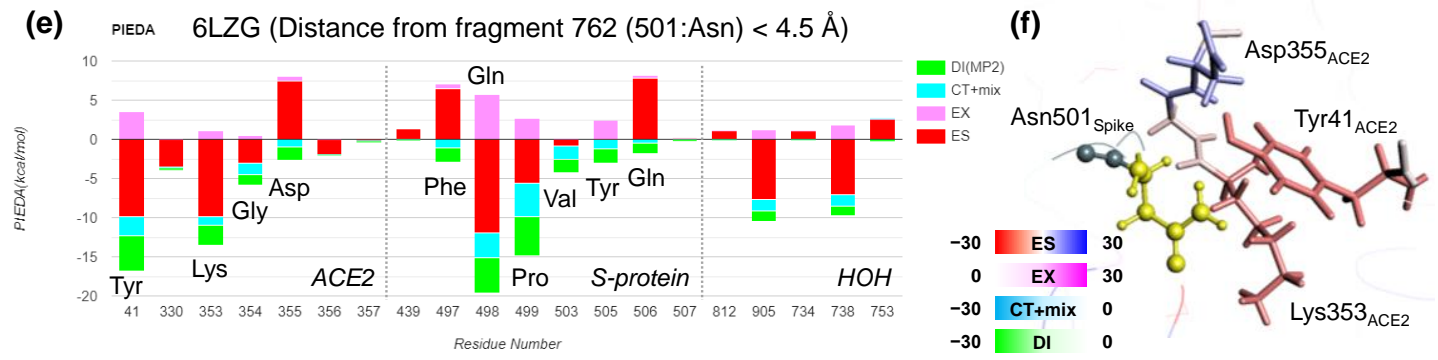

Figure 15. FMO results for PPI between S protein RBD and ACE2 receptor (PDB ID: 6LZG、FMODB ID: 4NZVN). (a) PIEDA main component between the ACE2 (yellow) and residue fragments of RBD, mapped on the molecular structure of RBD using a BioStation Viewer. (b) PIEDA main component between the RBD (yellow) and residue fragments of ACE2, mapped on the molecular structure of ACE2. (c) Graphical representation of the PIEDA components between the Lys417 of S protein and a residue fragment within 4.5 Å using the FMODB web interface. (d) PIEDA main component as in (c), mapped on the molecular structure. (e) Graphical representation of the PIEDA components between the Asn501 of S protein (yellow) and a residue fragment within $4.5 \AA$, using the FMODB web interface. (f) PIEDA main component as in (b), mapped on the molecular structure. Energy values are in $\mathrm{kcal} / \mathrm{mol}$.

As an example of RBD-neutralizing antibodies, we show the FMO results for a structure containing BD-629 Fab, which has many overlapping active sites on RBD with the ACE2 receptor and a high activity value ( $\mathrm{IC}_{50}$ ) (PDB ID: 7CH5, FMODB ID: JM5M9) ${ }^{50}$. First, the main components of PIEDA for the PPI of RBD and BD-629 Fab were mapped on the molecular structure (Figures 16a,b). ES is the main component of many interactions between RBD and BD-629 Fab, whereas only Tyr52 $2_{\mathrm{H}}$, Tyr99 ${ }_{\mathrm{H}}$, and Phe32 $2_{\mathrm{L}}$ of BD-629 Fab interact 
with RBD by DI as the main component (subscripts $\mathrm{L}$ and $\mathrm{H}$ indicate heavy and light chains of the antibody, respectively). Next, PIEDA on a single residue of Lys417 (Figure 16c,d) showed that $\mathrm{Asp} 101_{\mathrm{H}}$ and $\mathrm{Tyr} 102_{\mathrm{H}}$ exhibit strong ES interactions. In particular, a salt bridge is formed between Lys417 and Asp101 $1_{\mathrm{H}}$, and the ES term is about $-150 \mathrm{kcal} / \mathrm{mol}$. In addition, $\mathrm{Tyr} 52_{\mathrm{H}}$ forms a $\mathrm{CH} / \pi$ interaction with the Lys417 side chain, and the DI term is the major component. On the other hand, considering Asn501 (Figures 16e,f), hydrogen bonds are formed between Asn501 and Ser30 $\mathrm{L}$, indicating a strong ES interaction. These results suggest that Lys417 and Asn501, which are characteristic of South African and Brazilian mutants, contribute significantly to the interaction with neutralizing antibodies similar to the ACE2 receptor. Details of the epitope analysis by neutralizing antibodies ${ }^{50}$ and mutant strains ${ }^{51}$ are available in the respective references.

(a)

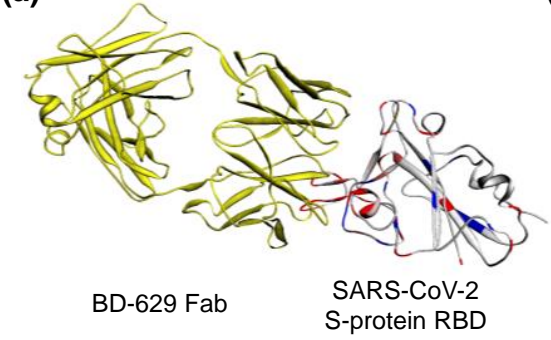

(b)

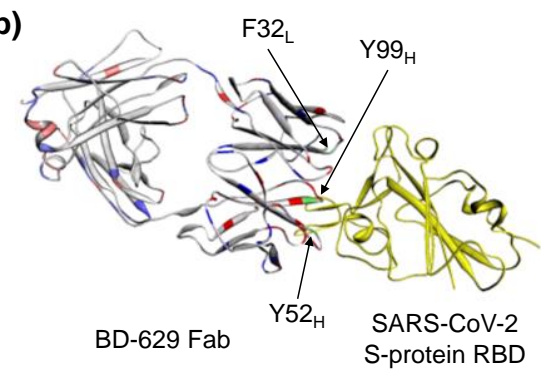

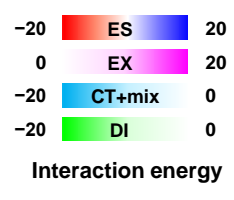

(d)

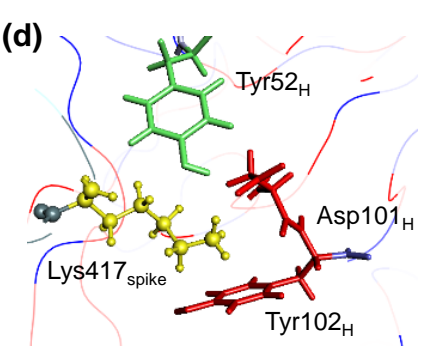

(f)

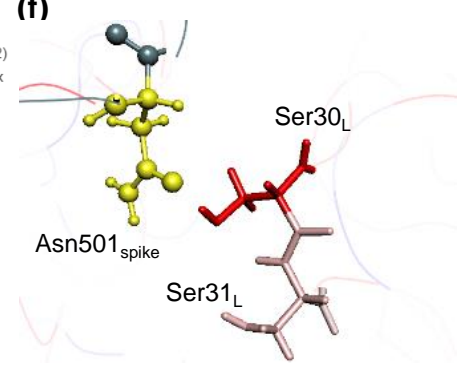

Figure 16. FMO results for PPI between S protein RBD and neutralizing antibody (PDB ID: 7CH5,

FMODB ID: JM5M9). (a) PIEDA main component between the BD-629 Fab (yellow) and residue fragments of RBD, mapped on the molecular structure of RBD using a BioStation Viewer. (b) PIEDA main component between the RBD (yellow) and residue fragments of BD-629 Fab, mapped on the molecular 
structure of BD-629 Fab. (c) Graphical representation of the PIEDA components between the Lys417 of S protein and a residue fragment within $4.5 \AA$ using the FMODB web interface. (d) PIEDA main component as in (c), mapped on the molecular structure. (e) Graphical representation of the PIEDA components between the Asn501 of S protein (yellow) and a residue fragment within $4.5 \AA$, using the FMODB web interface. (f) PIEDA main component as in (b), mapped on the molecular structure. Energy values are in $\mathrm{kcal} / \mathrm{mol}$.

\subsection{Nucleocapsid (N) protein}

The nucleocapsid (N) protein, a SP, binds to viral genomic RNA to form a capsid, which constitutes the viral particle. Both the $\mathrm{N}$-terminal domain (NTD) and $\mathrm{C}$-terminal domain (CTD) are registered in $\mathrm{PDB}^{55}$. NTD is a major RNA-binding domain, and its complex structure with single- and double-stranded RNA has been determined by solution NMR ${ }^{56}$. CTD is the dimerization domain, and its dimeric structure has been obtained by X-ray crystallography ${ }^{57,58}$, suggesting that it also has an RNA-binding ability. The calculation results of these structures are registered in FMODB, and representative analysis of the complex structure of NTD and single-stranded RNA (PDBID:7ACT, FMODB ID: N3Y7Q) is described below. Figure 17a shows the interaction of each amino acid residue of $\mathrm{N}$ protein from RNA, mapping the main components of PIEDA on the molecular structure. We confirmed that the molecular recognition of RNA is mainly due to ES interactions. On the other hand, Figure 17b shows the DI interaction between the protein and only the base parts of RNA, indicating that DI plays an important role in recognizing the bases. For example, a large DI is observed in the interaction between the U3 base and Arg95, indicating $\pi-\pi$ stacking between the pyrimidine of U3 and guanidyl group of Arg95 (Figures 17c,d). Figure 17e shows the structure of the NTD with and without RNA binding (PDDBID: 7CDZ, FMODB ID: R12G8). As shown in Figure 17f, the interaction energy between the red-framed loop (Arg92-Ser105) and RNA is $-1191.5 \mathrm{kcal} / \mathrm{mol}$, which accounts for $53.8 \%$ of the total interaction. This indicates that the structural change is important in the binding of $\mathrm{N}$ protein to RNA. 


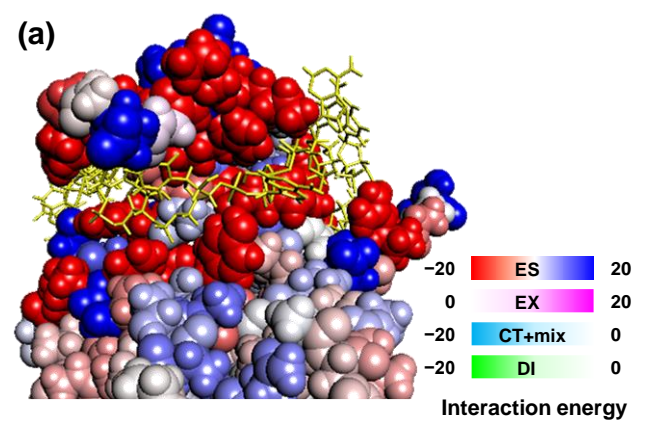

(b)

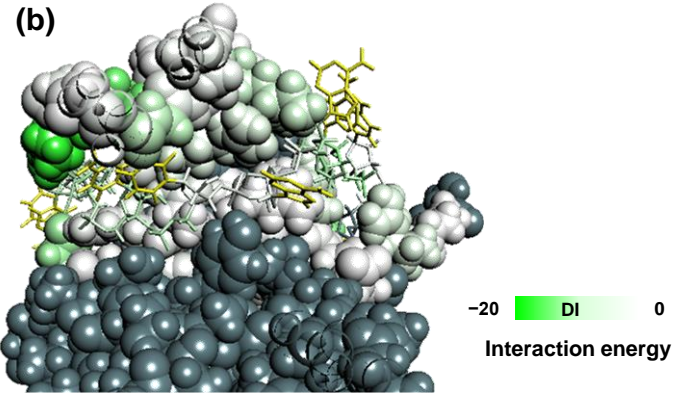

(c)

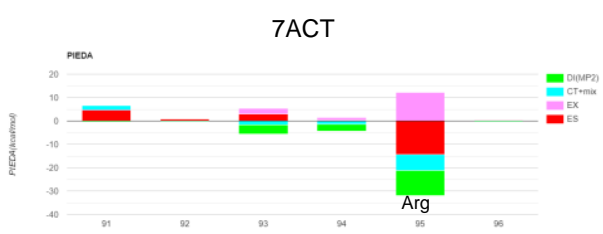

(d)

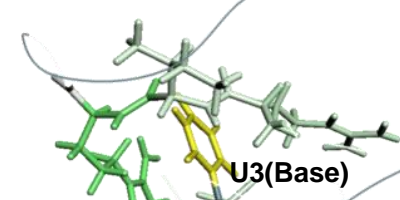

(e)

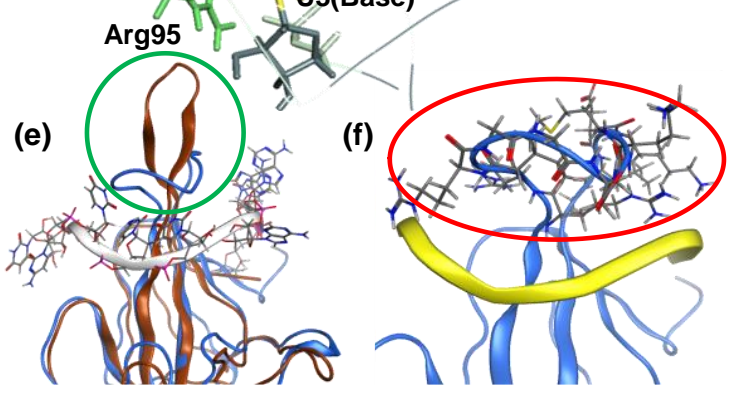

Figure 17. FMO results for $\mathrm{N}$ protein and single-stranded RNA (PDDBID:7CDZ, FMODB ID: R12G8) . Energies are in $\mathrm{kcal} / \mathrm{mol}$. (a) PIEDA main component between the RNA (yellow) and residue fragments of

$\mathrm{N}$ protein, mapped on the molecular structure of $\mathrm{N}$ protein using a BioStation Viewer. (b) PIEDA DI component between the RNA (yellow) and residue fragments of N protein. (c) Graphical representation of the PIEDA components between the U3 base of RNA and a residue fragment within $4.5 \AA$ using the FMODB web interface. (d) PIEDA main component as in (c), mapped on the molecular structure. (e) the structure of the NTD with (blue) and without (brown) RNA binding. (f) Arg92-Ser105 are framed by red loop. Energy values are in $\mathrm{kcal} / \mathrm{mol}$.

\subsection{Other SARS-CoV-2}

Structures other than the above nine are currently classified as Other SARS-CoV-2. Although several cryo-EM structures of nsp1, which suppress the innate immune function of the host, in a huge complex with human $40 \mathrm{~S}$ ribosomal subunit have been published in $\mathrm{PDB}^{59}$, ${ }^{60}$, FMODB currently has only the calculated X-ray crystal structure of nsp1 alone (PDBID 7K7P, FMODBID: 5965Z) ${ }^{61}$, and the complex structure is under calculation. The structures of nsp2 (PDBID: 7MSW) and nsp14 (PDBID: 7DIY), which were published in PDB in May 2021, are currently under investigation. For SP, a five-helix bundle structure of Envelope (E) protein $^{62}$ (PDBID: 7K3G, FMODB ID: 4NZYN) and a complex structure of E protein Cterminal DRLV motif and human cell junction protein PALS163 (PDBID: 7M4R, FMODB ID: Z2JJN) have been registered in FMODB. For Open reading Form proteins, FMO calculation results for ORF3a (PDBID: 6XDC), ORF7a (PDBID: 7CI3), ORF8 (PDBID: 
7JX6), OLF9b (PDBID: 6Z4U), ORF9b-Human Tom70 (Mitochondrial import receptor subunit) complex (PDBID: 7KDT) ${ }^{64}$ have been registered in FMODB.

\section{Conclusion}

In this study, we present an overview of the COVID-19 special feature in FMODB, describing the contents of the registered data and analysis methods for each type of SARSCoV-2 related protein. We have collected data mainly on the structures classified as representative entries in the COVID-19 special page of $\mathrm{PDBj}$, but it is difficult to completely cover them due to some technical issues. First, regarding the handling of $\mathrm{Zn}$ atoms in PLpro, RdRp, Helicase, nsp10, and ACE2, only a few of the Zn-containing proteins have been currently calculated in the presence of $\mathrm{Zn}$ because of the large amount of manual work involved in fragmentation. Since $\mathrm{Zn}$ is located far from the active site in these proteins, its presence or absence would not affect important interactions. Automatic fragmentation around $\mathrm{Zn}$ is currently under development and will be recalculated and registered in FMODB as soon as it is available. Furthermore, for S glycoproteins containing carbohydrate chains, we have calculated structural models with and without carbohydrate modification and registered them in FMODB (e.g., PDBID: 6LZG, FMODBID: 4NZVN and YQG92). The third issue is the size of the calculated protein. Currently, complex structures of up to 1000 residues are registered in FMODB (7BV2, 1057 fragment). Among the FMO calculations performed by ABINIT-MP so far, the largest-sized protein is the trimer of $\mathrm{S}$ protein $(3,300 \text { residues })^{48}$, which is currently under preparation for registration for both open and closed structures. In the near future, we shall perform FMO calculations for large complex systems, such as RTCs of RdRp, including Helicase, nsp1-robosomal subunit, and Spike trimer-antibody, and elucidate the molecular recognition mechanism in these complex systems. We shall address these technical issues in the activities of the FMO drug design consortium (FMODD) ${ }^{65}$.

Although the current FMODB includes only results calculated using the ABINIT-MP program ${ }^{18,19}$, some groups have performed FMO calculations for COVID-19-related proteins, such as Mpro ${ }^{66} 67$ and S protein ${ }^{68}$, using the GAMESS program ${ }^{17} 69,70$. In particular, the FMODFTB method included in GAMESS is suitable for screening owing to its much lower computational cost ${ }^{71}$. FMO-DFTB is also employed as a part of the in silico drug discovery pipeline using supercomputers ${ }^{72}$. We currently improve FMODB so that the results of FMO calculations can be registered in GAMESS.

Experimental structural studies of SARS-CoV-2 by structural biologists worldwide and their publications in the PDB are a valuable source of information for the fight against COVID-19. The interaction data published in FMODB enhance our understanding of SARS-CoV-2 by 
adding quantitative interaction to structural information. Clarifying structural features of therapeutic agents important to protein interactions would not only help in the rational design of therapeutic agents but also contribute to the epitope analysis of mutant strains, which is currently the focus of global attention, and the identification of interactions leading to vaccine development. We shall continuously update the FMODB by performing FMO calculations on newly released important PDB structures, which we hope will help overcome COVID-19.

\section{Acknowledgement}

We thank Prof. Yuji Mochizuki and Dr. Koji Okuwaki of Rikkyo University, Prof. Shigenori Tanaka of Kobe University, Dr. Yuto Komeiji of National Institute of Advanced Industrial Science and Technology, and Dr. Tatsuya Nakano and Dr. Yoshio Okiyama of National institute of health sciences (NIHS) for general discussions related to FMO calculations. In particular, Prof. Mochizuki provided us the Oakforest-PACS version of ABINIT-MP and the calculation data of the spike protein trimer, which is in preparation for registration. We are grateful to Prof. Tatsuya Takagi of Osaka University for discussing the PPI of S protein, Dr. Kazumi Tsuda and Dr. Shu Koyama of Science \& Technology Systems, Inc. for their help in the development of FMOBD, and Dr. Junichi Ishida of Mizuho Research \& Technologies, Ltd. for his help in some of the calculations.

This study was partially supported by the Platform Project for Supporting Drug Discovery and Life Science Research (Basis for Supporting Innovative Drug Discovery and Life Science Research) (BINDS) from the Japan Agency for Medical Research and Development (AMED) (grant number JP21am0101113). CW acknowledges JST, PRESTO grant (JPMJPR18GD). CW and DT acknowledge JSPS KAKENHI Grant No. 18K06619. This research was supported by the RIKEN programs for Drug Discovery and Medical Technology Platforms (DMP) and COVID-19 project. This research was performed in the activities of the FMO drug design consortium (FMODD). The FMO calculations were partially performed using the OakforestPACS supercomputer (project ID: hp200101), FUGAKU supercomputer (project ID: hp210130), the HOKUSAI supercomputer (RIKEN Advanced Center for Computing and Communications, Saitama, Japan, project ID: Q20306), and the TSUBAME3.0 supercomputer (Tokyo Institute of Technology, Tokyo, Japan). A part of PIEDA calculations were performed with the MIZUHO/BioStation software package (version 4.0).

\section{References}

1. WHO Coronavirus disease (COVID-19) situation reports. 
https://www.who.int/emergencies/diseases/novel-coronavirus-2019/situation-reports/

2. Burley, S. K.; Berman, H. M.; Bhikadiya, C.; Bi, C.; Chen, L.; Costanzo, L. D.; Christie, C.; Duarte, J. M.; Dutta, S.; Feng, Z.; Ghosh, S.; Goodsell, D. S.; Green, R. K.; Guranovic, V.; Guzenko, D.; Hudson, B. P.; Liang, Y.; Lowe, R.; Peisach, E.; Periskova, I.; Randle, C.; Rose, A.; Sekharan, M.; Shao, C.; Tao, Y.-P.; Valasatava, Y.; Voigt, M.; Westbrook, J.; Young, J.; Zardecki, C.; Zhuravleva, M.; Kurisu, G.; Nakamura, H.; Kengaku, Y.; Cho, H.; Sato, J.; Kim, J. Y.; Ikegawa, Y.; Nakagawa, A.; Yamashita, R.; Kudou, T.; Bekker, G.-J.; Suzuki, H.; Iwata, T.; Yokochi, M.; Kobayashi, N.; Fujiwara, T.; Velankar, S.; Kleywegt, G. J.; Anyango, S.; Armstrong, D. R.; Berrisford, J. M.; Conroy, M. J.; Dana, J. M.; Deshpande, M.; Gane, P.; Gáborová, R.; Gupta, D.; Gutmanas, A.; Koča, J.; Mak, L.; Mir, S.; Mukhopadhyay, A.; Nadzirin, N.; Nair, S.; Patwardhan, A.; Paysan-Lafosse, T.; Pravda, L.; Salih, O.; Sehnal, D.; Varadi, M.; Vařeková, R.; Markley, J. L.; Hoch, J. C.; Romero, P. R.; Baskaran, K.; Maziuk, D.; Ulrich, E. L.; Wedell, J. R.; Yao, H.; Livny, M.; Ioannidis, Y. E., Protein Data Bank: the single global archive for 3D macromolecular structure data. Nucleic Acids Research 2019, 47, D520-D528.

3. Waman, V. P.; Sen, N.; Varadi, M.; Daina, A.; Wodak, S. J.; Zoete, V.; Velankar, S.; Orengo, C., The impact of structural bioinformatics tools and resources on SARS-CoV-2 research and therapeutic strategies. Brief Bioinform 2021, 22, 742-768.

4. Kinjo, A. R.; Bekker, G.-J.; Suzuki, H.; Tsuchiya, Y.; Kawabata, T.; Ikegawa, Y.; Nakamura, H., Protein Data Bank Japan (PDBj): updated user interfaces, resource description framework, analysis tools for large structures. Nucleic Acids Research 2017, 45, D282-D288.

5. Kinjo, A. R., Bekker, G.-J., Wako, H., Endo, S., Tsuchiya, Y., Sato, H., Nishi, H., Kinoshita, K., Suzuki, H., Kawabata, T., Yokochi, M., Iwata, T., Kobayashi, N., Fujiwara, T., Kurisu, G. and Nakamura, H., New tools and functions in Data-out activities at Protein Data Bank Japan (PDBj). Protein Science 2018, 27, 95-102.

6. Kitaura et. al., Fragment molecular orbital method: an approximate computational method for large molecules. chemical physics letters 1999, 313, 701-706.

7. Yuji Mochizuki, S. T., Kaori Fukuzawa, eds., Recent Advances of the Fragment Molecular Orbital Method. Springer, Singapore: 2021.

8. Takaya, D.; Watanabe, C.; Nagase, S.; Kamisaka, K.; Okiyama, Y.; Moriwaki, H.; Yuki, H.; Sato, T.; Kurita, N.; Yagi, Y.; Takagi, T.; Kawashita, N.; Takaba, K.; Ozawa, T.; TakimotoKamimura, M.; Tanaka, S.; Fukuzawa, K.; Honma, T., FMODB: The World's First Database of Quantum Mechanical Calculations for Biomacromolecules Based on the Fragment Molecular Orbital Method. Journal of Chemical Information and Modeling 2021.

9. Watanabe, C.; Watanabe, H.; Okiyama, Y.; Takaya, D.; Fukuzawa, K.; Tanaka, S.; Honma, T., Development of an automated fragment molecular orbital (FMO) calculation protocol toward construction of quantum mechanical calculation database for large biomolecules. Chem- 
Bio Informatics Journal 2019, 19, 5-18.

10. BioStation Viewer, Open 1.0 rev. 23 (https://fmodd.jp/biostationviewer-dl/); FMODD consortium: Tokyo, Japan, 2021.

11. Molecular Operating Environment (MOE), 2019.01; Chemical Computing Group ULC: 1010 Sherbooke St. West, Suite \#910, Montreal, QC, Canada, H3A 2R7, 2021.

12. Fukuzawa, K.; Watanabe, C.; Okiyama, Y.; Nakano, T. How to Perform FMO Calculation in Drug Discovery. In Recent Advances of the Fragment Molecular Orbital Method, Mochizuki, Y.; Tanaka, S.; Fukuzawa, K., Eds.; Springer: Singapore, 2021.

13. Mochizuki, Y.; Nakano, T.; Koikegami, S.; Tanimori, S.; Abe, Y.; Nagashima, U.; Kitaura, K., A parallelized integral-direct second-order Moller-Plesset perturbation theory method with a fragment molecular orbital scheme. Theoretical Chemistry Accounts 2004, 112, 442-452.

14. Mochizuki, Y.; Koikegami, S.; Nakano, T.; Amari, S.; Kitaura, K., Large scale MP2 calculations with fragment molecular orbital scheme. Chemical Physics Letters 2004, 396, 473479 .

15. Fedorov, D. G.; Kitaura, K., Pair interaction energy decomposition analysis. J Comput Chem 2007, 28, 222-37.

16. Tsukamoto, T.; Kato, K.; Kato, A.; Nakano, T.; Mochizuki, Y.; Fukuzawa, K., Implementation of Pair Interaction Energy DecompositionAnalysis and Its Applications to Protein-Ligand Systems. Journal of Computer Chemistry, Japan 2015, 14, 1-9.

17. Heifetz, A.; James, T.; Southey, M.; Bodkin, M. J.; Bromidge, S. Guiding Medicinal Chemistry with Fragment Molecular Orbital (FMO) Method. In Quantum Mechanics in Drug Discovery, Heifetz, A., Ed.; Humana: New York, NY, 2020; Vol. 2114, Chapter 3, pp 37-48.

18. Mochizuki, Y.; Nakano, T.; Sakakura, K.; Okiyama, Y.; Watanabe, H.; Kato, K.; Akinaga, Y.; Sato, S.; Yamamoto, J.-i.; Yamashita, K.; Murase, T.; Ishikawa, T.; Komeiji, Y.; Kato, Y.; Watanabe, N.; Tsukamoto, T.; Mori, H.; Okuwaki, K.; Tanaka, S.; Kato, A.; Watanabe, C.; Fukuzawa, K. The ABINIT-MP Program. In Recent Advances of the Fragment Molecular Orbital Method, Mochizuki, Y.; Tanaka, S.; Fukuzawa, K., Eds.; Springer: Singapore, 2021, pp 53-67.

19. Tanaka, S.; Mochizuki, Y.; Komeiji, Y.; Okiyama, Y.; Fukuzawa, K., Electron-correlated fragment-molecular-orbital calculations for biomolecular and nano systems. Phys Chem Chem Phys 2014, 16, 10310-44.

20. Barnes, C. O.; West, A. P.; Huey-Tubman, K. E.; Hoffmann, M. A. G.; Sharaf, N. G.; Hoffman, P. R.; Koranda, N.; Gristick, H. B.; Gaebler, C.; Muecksch, F.; Lorenzi, J. C. C.; Finkin, S.; Hägglöf, T.; Hurley, A.; Millard, K. G.; Weisblum, Y.; Schmidt, F.; Hatziioannou, T.; Bieniasz, P. D.; Caskey, M.; Robbiani, D. F.; Nussenzweig, M. C.; Bjorkman, P. J., Structures of Human Antibodies Bound to SARS-CoV-2 Spike Reveal Common Epitopes and Recurrent Features of Antibodies. Cell 2020, 182, 828-842.e16. 
21. Walls, A. C.; Park, Y. J.; Tortorici, M. A.; Wall, A.; McGuire, A. T.; Veesler, D., Structure, Function, and Antigenicity of the SARS-CoV-2 Spike Glycoprotein. Cell 2020, 181, 281-292 e6.

22. Hilgenfeld, R., From SARS to MERS: crystallographic studies on coronaviral proteases enable antiviral drug design. FEBS J 2014, 281, 4085-96.

23. Yan, L.; Ge, J.; Zheng, L.; Zhang, Y.; Gao, Y.; Wang, T.; Huang, Y.; Yang, Y.; Gao, S.; Li, M.; Liu, Z.; Wang, H.; Li, Y.; Chen, Y.; Guddat, L. W.; Wang, Q.; Rao, Z.; Lou, Z., Cryo-EM Structure of an Extended SARS-CoV-2 Replication and Transcription Complex Reveals an Intermediate State in Cap Synthesis. Cell 2021, 184, 184-193.e10.

24. Lim, K. P.; Ng, L. F. P.; Liu, D. X., Identification of a Novel Cleavage Activity of the First Papain-Like Proteinase Domain Encoded by Open Reading Frame 1a of the Coronavirus Avian Infectious Bronchitis Virus and Characterization of the Cleavage Products. Journal of Virology 2000, 74, 1674-1685.

25. Harcourt, B. H.; Jukneliene, D.; Kanjanahaluethai, A.; Bechill, J.; Severson, K. M.; Smith, C. M.; Rota, P. A.; Baker, S. C., Identification of Severe Acute Respiratory Syndrome Coronavirus Replicase Products and Characterization of Papain-Like Protease Activity. Journal of Virology 2004, 78, 13600-13612.

26. Gao, X.; Qin, B.; Chen, P.; Zhu, K.; Hou, P.; Wojdyla, J. A.; Wang, M.; Cui, S., Crystal structure of SARS-CoV-2 papain-like protease. Acta Pharmaceutica Sinica B 2021, 11, 237-245.

27. Frieman, M.; Ratia, K.; Johnston, R. E.; Mesecar, A. D.; Baric, R. S., Severe Acute Respiratory Syndrome Coronavirus Papain-Like Protease Ubiquitin-Like Domain and Catalytic Domain Regulate Antagonism of IRF3 and NF- $\kappa$ B Signaling. Journal of Virology 2009, 83, 66896705.

28. Shin, D.; Mukherjee, R.; Grewe, D.; Bojkova, D.; Baek, K.; Bhattacharya, A.; Schulz, L.; Widera, M.; Mehdipour, A. R.; Tascher, G.; Geurink, P. P.; Wilhelm, A.; van der Heden van Noort, G. J.; Ovaa, H.; Muller, S.; Knobeloch, K. P.; Rajalingam, K.; Schulman, B. A.; Cinatl, J.; Hummer, G.; Ciesek, S.; Dikic, I., Papain-like protease regulates SARS-CoV-2 viral spread and innate immunity. Nature 2020, 587, 657-662.

29. Rut, W.; Lv, Z.; Zmudzinski, M.; Patchett, S.; Nayak, D.; Snipas, S. J.; El Oualid, F.; Huang, T. T.; Bekes, M.; Drag, M.; Olsen, S. K., Activity profiling and crystal structures of inhibitor-bound SARS-CoV-2 papain-like protease: A framework for anti-COVID-19 drug design. Sci Adv 2020, 6.

30. Michalska, K.; Kim, Y.; Jedrzejczak, R.; Maltseva, N. I.; Stols, L.; Endres, M.; Joachimiak, A., Crystal structures of SARS-CoV-2 ADP-ribose phosphatase: from the apo form to ligand complexes. IUCrJ 2020, 7, 814-824.

31. Jin, Z.; Du, X.; Xu, Y.; Deng, Y.; Liu, M.; Zhao, Y.; Zhang, B.; Li, X.; Zhang, L.; Peng, C.; Duan, Y.; Yu, J.; Wang, L.; Yang, K.; Liu, F.; Jiang, R.; Yang, X.; You, T.; Liu, X.; Yang, X.; Bai, 
F.; Liu, H.; Liu, X.; Guddat, L. W.; Xu, W.; Xiao, G.; Qin, C.; Shi, Z.; Jiang, H.; Rao, Z.; Yang, H., Structure of M(pro) from SARS-CoV-2 and discovery of its inhibitors. Nature 2020, 582, 289-293. 32. Hatada, R.; Okuwaki, K.; Mochizuki, Y.; Handa, Y.; Fukuzawa, K.; Komeiji, Y.; Okiyama, Y.; Tanaka, S., Fragment Molecular Orbital Based Interaction Analyses on COVID-19 Main Protease - Inhibitor N3 Complex (PDB ID: 6LU7). J Chem Inf Model 2020, 60, 3593-3602.

33. Hatada, R.; Okuwaki, K.; Akisawa, K.; Mochizuki, Y.; Handa, Y.; Fukuzawa, K.; Komeiji, Y.; Okiyama, Y.; Tanaka, S., Statistical interaction analyses between SARS-CoV-2 main protease and inhibitor N3 by combining molecular dynamics simulation and fragment molecular orbital calculation. Applied Physics Express 2021, 14.

34. Dai, W.; Zhang, B.; Jiang, X.-M.; Su, H.; Li, J.; Zhao, Y.; Xie, X.; Jin, Z.; Peng, J.; Liu, F.; Li, C.; Li, Y.; Bai, F.; Wang, H.; Cheng, X.; Cen, X.; Hu, S.; Yang, X.; Wang, J.; Liu, X.; Xiao, G.; Jiang, H.; Rao, Z.; Zhang, L.-K.; Xu, Y.; Yang, H.; Liu, H., Structure-based design of antiviral drug candidates targeting the SARS-CoV-2 main protease. Science 2020, 368, 1331-1335.

35. Yang, H.; Xie, W.; Xue, X.; Yang, K.; Ma, J.; Liang, W.; Zhao, Q.; Zhou, Z.; Pei, D.; Ziebuhr, J.; Hilgenfeld, R.; Yuen, K. Y.; Wong, L.; Gao, G.; Chen, S.; Chen, Z.; Ma, D.; Bartlam, M.; Rao, Z., Design of wide-spectrum inhibitors targeting coronavirus main proteases. PLoS Biol 2005, 3, e324.

36. Kurauchi, R.; Watanabe, C.; Fukuzawa, K.; Tanaka, S., Novel type of virtual ligand screening on the basis of quantum-chemical calculations for protein-ligand complexes and extended clustering techniques. Computational and Theoretical Chemistry 2015, 1061, 12-22.

37. Ma, C.; Sacco, M. D.; Hurst, B.; Townsend, J. A.; Hu, Y.; Szeto, T.; Zhang, X.; Tarbet, B.; Marty, M. T.; Chen, Y.; Wang, J., Boceprevir, GC-376, and calpain inhibitors II, XII inhibit SARS-CoV-2 viral replication by targeting the viral main protease. Cell Res 2020, 30, 678-692.

38. Hillen, H. S.; Kokic, G.; Farnung, L.; Dienemann, C.; Tegunov, D.; Cramer, P., Structure of replicating SARS-CoV-2 polymerase. Nature 2020, 584, 154-156.

39. Yin, W.; Mao, C.; Luan, X.; Shen, D. D.; Shen, Q.; Su, H.; Wang, X.; Zhou, F.; Zhao, W.; Gao, M.; Chang, S.; Xie, Y. C.; Tian, G.; Jiang, H. W.; Tao, S. C.; Shen, J.; Jiang, Y.; Jiang, H.; Xu, Y.; Zhang, S.; Zhang, Y.; Xu, H. E., Structural basis for inhibition of the RNA-dependent RNA polymerase from SARS-CoV-2 by remdesivir. Science 2020, 368, 1499-1504.

40. Kato, K.; Honma, T.; Fukuzawa, K., Intermolecular interaction among Remdesivir, RNA and RNA-dependent RNA polymerase of SARS-CoV-2 analyzed by fragment molecular orbital calculation. J Mol Graph Model 2020, 100, 107695.

41. Chen, J.; Malone, B.; Llewellyn, E.; Grasso, M.; Shelton, P. M. M.; Olinares, P. D. B.; Maruthi, K.; Eng, E. T.; Vatandaslar, H.; Chait, B. T.; Kapoor, T. M.; Darst, S. A.; Campbell, E. A., Structural Basis for Helicase-Polymerase Coupling in the SARS-CoV-2 ReplicationTranscription Complex. Cell 2020, 182, 1560-1573 e13. 
42. Nudler, E., RNA polymerase backtracking in gene regulation and genome instability. Cell 2012, 149, 1438-45.

43. Kaplan, D. L.; O'Donnell, M., DnaB Drives DNA Branch Migration and Dislodges Proteins While Encircling Two DNA Strands. Molecular Cell 2002, 10, 647-657.

44. Hackbart, M.; Deng, X.; Baker, S. C., Coronavirus endoribonuclease targets viral polyuridine sequences to evade activating host sensors. Proc Natl Acad Sci US A 2020, 117, 80948103.

45. Kim, Y.; Wower, J.; Maltseva, N.; Chang, C.; Jedrzejczak, R.; Wilamowski, M.; Kang, S.; Nicolaescu, V.; Randall, G.; Michalska, K.; Joachimiak, A., Tipiracil binds to uridine site and inhibits Nsp15 endoribonuclease NendoU from SARS-CoV-2. Communications Biology 2021, 4. 46. Lin, S.; Chen, H.; Ye, F.; Chen, Z.; Yang, F.; Zheng, Y.; Cao, Y.; Qiao, J.; Yang, S.; Lu, G., Crystal structure of SARS-CoV-2 nsp10/nsp16 2'-O-methylase and its implication on antiviral drug design. Signal Transduct Target Ther 2020, 5, 131.

47. Rosas-Lemus, M.; Minasov, G.; Shuvalova, L.; Inniss, N. L.; Kiryukhina, O.; Brunzelle, J.; Satchell, K. J. F., High-resolution structures of the SARS-CoV-2 2' -O-methyltransferase reveal strategies for structure-based inhibitor design. Science Signaling 2020, 13, eabe1202.

48. Akisawa, K.; Hatada, R.; Okuwaki, K.; Mochizuki, Y.; Fukuzawa, K.; Komeiji, Y.; Tanaka, S., Interaction analyses of SARS-CoV-2 spike protein based on fragment molecular orbital calculations. RSC Advances 2021, 11, 3272-3279.

49. Watanabe, C.; Okiyama, Y.; Tanaka, S.; Fukuzawa, K.; Honma, T., Molecular recognition of SARS-CoV-2 spike glycoprotein: quantum chemical hot spot and epitope analyses. Chemical Science 2021.

50. Watanabe, K.; Watanabe, C.; Honma, T.; Tian, Y.-S.; Kawashima, Y.; Kawashita, N.; Takagi, T.; Fukuzawa, K., Intermolecular Interaction Analyses on SARS-CoV-2 Spike Protein Receptor Binding Domain and Human Angiotensin-Converting Enzyme 2 Receptor-Blocking Antibody/Peptide Using Fragment Molecular Orbital Calculation. J. Phys. Chem. Lett. 2021, 12. 51. Kazuki Watanabe, C. W., Teruki Honma, Yu-Shi Tian, Yusuke Kawashima, Norihito Kawashita, Kaori Fukuzawa, Tatsuya Takagi, Computational ab initio interaction analyses between neutralizing antibody and SARS-CoV-2 variant spike proteins using the fragment molecular orbital method. Bull Chem Soc Jpn 2021.

52. Kirby, T., New variant of SARS-CoV-2 in UK causes surge of COVID-19. The Lancet Respiratory Medicine 2021, 9, e20-e21.

53. Tegally, H.; Wilkinson, E.; Giovanetti, M.; Iranzadeh, A.; Fonseca, V.; Giandhari, J.; Doolabh, D.; Pillay, S.; San, E. J.; Msomi, N.; Mlisana, K.; Von Gottberg, A.; Walaza, S.; Allam, M.; Ismail, A.; Mohale, T.; Glass, A. J.; Engelbrecht, S.; Van Zyl, G.; Preiser, W.; Petruccione, F.; Sigal, A.; Hardie, D.; Marais, G.; Hsiao, M.; Korsman, S.; Davies, M.-A.; Tyers, L.; Mudau, I.; 
York, D.; Maslo, C.; Goedhals, D.; Abrahams, S.; Laguda-Akingba, O.; Alisoltani-Dehkordi, A.; Godzik, A.; Wibmer, C. K.; Sewell, B. T.; Lourenço, J.; Alcantara, L. C. J.; Pond, S. L. K.; Weaver, S.; Martin, D.; Lessells, R. J.; Bhiman, J. N.; Williamson, C.; De Oliveira, T., In; Cold Spring Harbor Laboratory: 2020.

54. Voloch, C. M.; Da Silva Francisco, R.; De Almeida, L. G. P.; Cardoso, C. C.; Brustolini, O. J.; Gerber, A. L.; Guimarães, A. P. D. C.; Mariani, D.; Da Costa, R. M.; Ferreira, O. C.; Frauches, T. S.; De Mello, C. M. B.; Leitão, I. D. C.; Galliez, R. M.; Faffe, D. S.; Castiñeiras, T. M. P. P.; Tanuri, A.; De Vasconcelos, A. T. R., Genomic characterization of a novel SARS-CoV-2 lineage from Rio de Janeiro, Brazil. Journal of Virology 2021.

55. Peng, Y.; Du, N.; Lei, Y.; Dorje, S.; Qi, J.; Luo, T.; Gao, G. F.; Song, H., Structures of the SARS - CoV - 2 nucleocapsid and their perspectives for drug design. The EMBO Journal 2020, 39, e105938.

56. Dinesh, D. C.; Chalupska, D.; Silhan, J.; Koutna, E.; Nencka, R.; Veverka, V.; Boura, E., Structural basis of RNA recognition by the SARS-CoV-2 nucleocapsid phosphoprotein. PLOS Pathogens 2020, 16, e1009100.

57. Zinzula, L.; Basquin, J.; Bohn, S.; Beck, F.; Klumpe, S.; Pfeifer, G.; Nagy, I.; Bracher, A.; Hartl, F. U.; Baumeister, W., High-resolution structure and biophysical characterization of the nucleocapsid phosphoprotein dimerization domain from the Covid-19 severe acute respiratory syndrome coronavirus 2. Biochem Biophys Res Commun 2021, 538, 54-62.

58. Y Yang, M.; He, S.; Chen, X.; Huang, Z.; Zhou, Z.; Zhou, Z.; Chen, Q.; Chen, S.; Kang, a. S., Structural Insight Into the SARS-CoV-2 Nucleocapsid Protein C-Terminal Domain Reveals a Novel Recognition Mechanism for Viral Transcriptional Regulatory Sequences. Front. Chem 2021, $12,1-12$

59. Thoms, M.; Buschauer, R.; Ameismeier, M.; Koepke, L.; Denk, T.; Hirschenberger, M.; Kratzat, H.; Hayn, M.; Mackens-Kiani, T.; Cheng, J.; Straub, J. H.; Sturzel, C. M.; Frohlich, T.; Berninghausen, O.; Becker, T.; Kirchhoff, F.; Sparrer, K. M. J.; Beckmann, R., Structural basis for translational shutdown and immune evasion by the Nsp1 protein of SARS-CoV-2. Science 2020, $369,1249-1255$.

60. Schubert, K.; Karousis, E. D.; Jomaa, A.; Scaiola, A.; Echeverria, B.; Gurzeler, L. A.; Leibundgut, M.; Thiel, V.; Muhlemann, O.; Ban, N., SARS-CoV-2 Nsp1 binds the ribosomal mRNA channel to inhibit translation. Nat Struct Mol Biol 2020, 27, 959-966.

61. Clark, L. K.; Green, T. J.; Petit, C. M., Structure of Nonstructural Protein 1 from SARSCoV-2. Journal of Virology 2021, 95, 1-12.

62. Mandala, V. S.; McKay, M. J.; Shcherbakov, A. A.; Dregni, A. J.; Kolocouris, A.; Hong, M., Structure and drug binding of the SARS-CoV-2 envelope protein transmembrane domain in lipid bilayers. Nat Struct Mol Biol 2020, 27, 1202-1208. 

A. S.; Obernier, K.; Guo, J. Z.; Batra, J.; Kaake, R. M.; Weckstein, A. R.; Owens, T. W.; Gupta, M.; Pourmal, S.; Titus, E. W.; Cakir, M.; Soucheray, M.; McGregor, M.; Cakir, Z.; Jang, G.; O'Meara, M. J.; Tummino, T. A.; Zhang, Z.; Foussard, H.; Rojc, A.; Zhou, Y.; Kuchenov, D.; Huttenhain, R.; Xu, J.; Eckhardt, M.; Swaney, D. L.; Fabius, J. M.; Ummadi, M.; Tutuncuoglu, B.; Rathore, U.; Modak, M.; Haas, P.; Haas, K. M.; Naing, Z. Z. C.; Pulido, E. H.; Shi, Y.; Barrio-Hernandez, I.; Memon, D.; Petsalaki, E.; Dunham, A.; Marrero, M. C.; Burke, D.; Koh, C.; Vallet, T.; Silvas, J. A.; Azumaya, C. M.; Billesbolle, C.; Brilot, A. F.; Campbell, M. G.; Diallo, A.; Dickinson, M. S.; Diwanji, D.; Herrera, N.; Hoppe, N.; Kratochvil, H. T.; Liu, Y.; Merz, G. E.; Moritz, M.; Nguyen, H. C.; Nowotny, C.; Puchades, C.; Rizo, A. N.; Schulze-Gahmen, U.; Smith, A. M.; Sun, M.; Young, I. D.; Zhao, J.; Asarnow, D.; Biel, J.; Bowen, A.; Braxton, J. R.; Chen, J.; Chio, C. M.; Chio, U. S.; Deshpande, I.; Doan, L.; Faust, B.; Flores, S.; Jin, M.; Kim, K.; Lam, V. L.; Li, F.; Li, J.; Li, Y. L.; Li, Y.; Liu, X.; Lo, M.; Lopez, K. E.; Melo, A. A.; Moss, F. R., 3rd; Nguyen, P.; Paulino, J.; Pawar, K. I.; Peters, J. K.; Pospiech, T. H., Jr.; Safari, M.; Sangwan, S.; Schaefer, K.; Thomas, P. V.; Thwin, A. C.; Trenker, R.; Tse, E.; Tsui, T. K. M.; Wang, F.; Whitis, N.; Yu, Z.; Zhang, K.; Zhang, Y.; Zhou, F.; Saltzberg, D.; Consortium, Q. S. B.; Hodder, A. J.; Shun-Shion, A. S.; Williams, D. M.; White, K. M.; Rosales, R.; Kehrer, T.; Miorin, L.; Moreno, E.; Patel, A. H.; Rihn, S.; Khalid, M. M.; Vallejo-Gracia, A.; Fozouni, P.; Simoneau, C. R.; Roth, T. L.; Wu, D.; Karim, M. A.; Ghoussaini, M.; Dunham, I.; Berardi, F.; Weigang, S.; Chazal, M.; Park, J.; Logue, J.; McGrath, M.; Weston, S.; Haupt, R.; Hastie, C. J.; Elliott, M.; Brown, F.; Burness, K. A.; Reid, E.; Dorward, M.; Johnson, C.; Wilkinson, S. G.; Geyer, A.; Giesel, D. M.; Baillie, C.; Raggett, S.; Leech, H.; Toth, R.; Goodman, N.; Keough, K. C.; Lind, A. L.; Zoonomia, C.; Klesh, R. J.; Hemphill, K. R.; Carlson-Stevermer, J.; Oki, J.; Holden, K.; Maures, T.; Pollard, K. S.; Sali, A.; Agard, D. A.; Cheng, Y.; Fraser, J. S.; Frost, A.; Jura, N.; Kortemme, T.; Manglik, A.; Southworth, D. R.; Stroud, R. M.; Alessi, D. R.; Davies, P.; Frieman, M. B.; Ideker, T.; Abate, C.; Jouvenet, N.; Kochs, G.; Shoichet, B.; Ott, M.; Palmarini, M.; Shokat, K. M.; Garcia-Sastre, A.; Rassen, J. A.; Grosse, R.; Rosenberg, O. S.; Verba, K. A.; Basler, C. F.; Vignuzzi, M.; Peden, A. A.; Beltrao, P.; Krogan, N. J., Comparative host-coronavirus protein interaction networks reveal pan-viral disease mechanisms. Science 2020, 370.

65. Fukuzawa, K.; Tanaka, S.; Yagi, Y.; Kurita, N.; Kawashita, N.; Takaba, K.; Honma, T. FMO Drug Design Consortium. In Recent Advances of the Fragment Molecular Orbital Method, Mochizuki, Y.; Tanaka, S.; Fukuzawa, K., Eds.; Springer: Singapore, 2021.

66. Nutho, B.; Mahalapbutr, P.; Hengphasatporn, K.; Pattaranggoon, N. C.; Simanon, N.; Shigeta, Y.; Hannongbua, S.; Rungrotmongkol, T., Why Are Lopinavir and Ritonavir Effective against the Newly Emerged Coronavirus 2019? Atomistic Insights into the Inhibitory Mechanisms. 
Biochemistry 2020, 59, 1769-1779.

67. Deetanya, P.; Hengphasatporn, K.; Wilasluck, P.; Shigeta, Y.; Rungrotmongkol, T.; Wangkanont, K., Interaction of 8-Anilinonaphthalene-1-Sulfonate with SARS-CoV-2 main protease and its application as a fluorescent probe for inhibitor identification. Computational and Structural Biotechnology Journal 2021.

68. Lim, H.; Baek, A.; Kim, J.; Kim, M. S.; Liu, J.; Nam, K. Y.; Yoon, J.; No, K. T., Hot spot profiles of SARS-CoV-2 and human ACE2 receptor protein protein interaction obtained by density functional tight binding fragment molecular orbital method. Sci Rep 2020, 10, 16862.

69. Fedorov, D. G., The fragment molecular orbital method: theoretical development, implementation in GAMESS , and applications. WIREs Computational Molecular Science 2017, 7, e1322.

70. Fedorov, D. G. Recent Development of the Fragment Molecular Orbital Method in GAMESS. In Recent Advances of the Fragment Molecular Orbital Method, Mochizuki, Y.; Tanaka, S.; Fukuzawa, K., Eds.; Springer: Singapore, 2021, pp 31-51.

71. Morao, I.; Heifetz, A.; Fedorov, D. G. Accurate Scoring in Seconds with the Fragment Molecular Orbital and Density-Functional Tight-Binding Methods. In Quantum Mechanics in Drug Discovery, Heifetz, A., Ed.; Humana: New York, NY, 2020; Vol. 2114, Chapter 9, pp 143 148.

72. Acharya, A.; Agarwal, R.; Baker, M. B.; Baudry, J.; Bhowmik, D.; Boehm, S.; Byler, K. G.; Chen, S. Y.; Coates, L.; Cooper, C. J.; Demerdash, O.; Daidone, I.; Eblen, J. D.; Ellingson, S.; Forli, S.; Glaser, J.; Gumbart, J. C.; Gunnels, J.; Hernandez, O.; Irle, S.; Kneller, D. W.; Kovalevsky, A.; Larkin, J.; Lawrence, T. J.; LeGrand, S.; Liu, S. H.; Mitchell, J. C.; Park, G.; Parks, J. M.; Pavlova, A.; Petridis, L.; Poole, D.; Pouchard, L.; Ramanathan, A.; Rogers, D. M.; Santos-Martins, D.; Scheinberg, A.; Sedova, A.; Shen, Y.; Smith, J. C.; Smith, M. D.; Soto, C.; Tsaris, A.; Thavappiragasam, M.; Tillack, A. F.; Vermaas, J. V.; Vuong, V. Q.; Yin, J.; Yoo, S.; Zahran, M.; Zanetti-Polzi, L., Supercomputer-Based Ensemble Docking Drug Discovery Pipeline with Application to Covid-19. J Chem Inf Model 2020, 60, 5832-5852. 
Table of Contents graphic
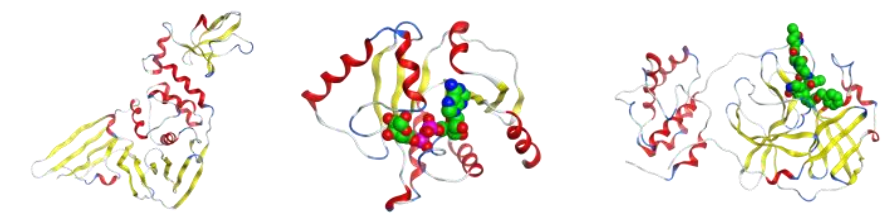

\section{COVID-19}
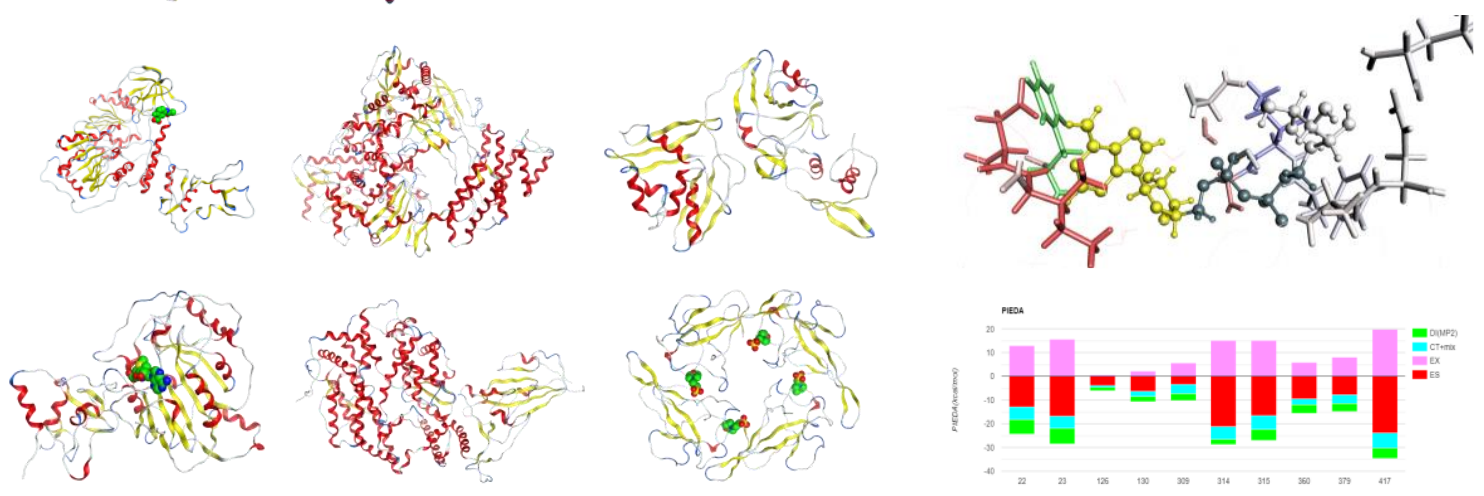